Supporting Information

\title{
Design, Synthesis, and Biological Evaluation of Potent Discodermolide Fluorescent and Photoaffinity Molecular Probes.
}

\footnotetext{
Amos B. Smith, III, ${ }^{1 *}$ Paul V. Rucker, ${ }^{1}$ Ignacio Brouard, ${ }^{1}$ B. Scott Freeze, ${ }^{1}$ Shujun Xia, ${ }^{2}$ and Susan Band Horwitz ${ }^{2}$

${ }^{1}$ Department of Chemistry, University of Pennsylvania, Philadelphia, PA 19104

${ }^{2}$ Department of Molecular Pharmacology, Albert Einstein College of Medicine, Bronx, NY, 10461
} 
Materials and Methods. Reactions were carried out in oven or flame-dried glassware under an argon atmosphere, unless otherwise noted. All solvents were reagent grade. Diethyl ether $\left(\mathrm{Et}_{2} \mathrm{O}\right)$ and tetrahydrofuran (THF) were freshly distilled from sodium/benzophenone under argon. $n$ butyllithium and $t$-butyllithium were purchased from Aldrich. Reactions were magnetically stirred and monitored by thin layer chromatography (TLC) with $0.25 \mathrm{~mm}$ E. Merck pre-coated silica gel plates. Flash chromatography was performed with silica gel 60 (particle size $0.040-0.062 \mathrm{~mm}$ ) supplied by Silicycle and Sorbent Technologies. Yields refer to chromatographically and spectroscopically pure compounds, unless otherwise stated. Infrared spectra were recorded on a Jasco Model FT/IR-480 Plus spectrometer. Proton and carbon-13 NMR spectra were recorded on a Bruker AMX-500 spectrometer. Chemical shifts are reported relative to chloroform ( $\delta 7.26)$, methanol $(\delta 3.31)$, acetonitrile $(\delta 1.94)$, DMSO $(\delta 2.50)$, or benzene $(\delta 7.15)$ for ${ }^{1} \mathrm{H}-\mathrm{NMR}$ and either chloroform ( $\delta 77.0)$, methanol $(\delta 49.2)$, acetonitrile $(\delta 118.7)$, DMSO $(\delta 39.5)$, or benzene $(\delta 128.0)$ for ${ }^{13} \mathrm{C}$ NMR. Optical rotations were measured on a Perkin-Elmer model 241 polarimeter. High resolution mass spectra were measured at the University of Pennsylvania Mass Spectrometry Service Center. 


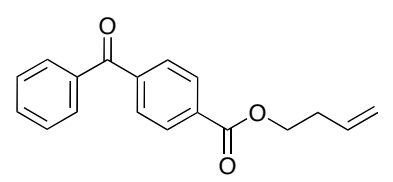

S1

Ketone S1: To a suspension of 4-benzoylbenzoic acid $(100 \mathrm{mg}, 0.442 \mathrm{mmol})$ in benzene (3.0 $\mathrm{mL}$ ) was added oxalyl chloride solution $\left(2.0 \mathrm{M} \mathrm{CH}_{2} \mathrm{Cl}_{2}, 0.5 \mathrm{~mL}, 1.0 \mathrm{mmol}\right)$, followed by 1 drop of DMF (dry, Aldrich Sure Seal ${ }^{\mathrm{TM}}$ ). After a vigorous evolution of gas, the reaction was allowed to stand for $5 \mathrm{~min}$. The reaction vessel was then heated to $43^{\circ} \mathrm{C}$ for $2 \mathrm{~h}$ and was subsequently allowed to cool to rt. The reaction contents were concentrated to a residue and were subsequently diluted with $0.3 \mathrm{~mL}$ of benzene and to the resulting solution was added 3-butene-1ol $(150 \mathrm{mg}, 2.1 \mathrm{mmol})$. The reaction mixture was maintained for an additonal $3 \mathrm{~h}$ and was quenched with $10 \mathrm{~mL}$ of saturated brine. The resulting emulsion was diluted with EtOAc $(100 \mathrm{~mL})$ and the bilayer was separated. The organic extract was further washed with water $(3 \times 30 \mathrm{~mL})$, dried $\left(\mathrm{Na}_{2} \mathrm{SO}_{4}\right)$, filtered and concentrated. The crude oil was purified by $\mathrm{SiO}_{2}$ column chromatography (3:1 hexanes/EtOAc) to furnish $60 \mathrm{mg} \mathrm{(50 \% )} \mathrm{of} \mathrm{olefin} \mathrm{S1.} \mathrm{IR} \mathrm{(film,} \mathrm{NaCl}$ ) 2926, 1717, 1653, 1269, $1102 \mathrm{~cm}^{-1} ;{ }^{1} \mathrm{H}$ NMR $\left(500 \mathrm{MHz}, \mathrm{CDCl}_{3}\right) \delta 8.14(\mathrm{~d}, J=8.2 \mathrm{~Hz}, 2 \mathrm{H}), 7.83(\mathrm{~d}, J=$ $8.2 \mathrm{~Hz}, 2 \mathrm{H}), 7.79(\mathrm{~d}, J=8.2 \mathrm{~Hz}, 2 \mathrm{H}), 7.61(\operatorname{app~t}, J=7.2 \mathrm{~Hz}, 1 \mathrm{H}), 7.49(\operatorname{app~t}, J=8.2 \mathrm{~Hz}, 2 \mathrm{H})$, $6.04(\mathrm{ddt}, J=16.4,10.2,7.0 \mathrm{~Hz}, 1 \mathrm{H}), 5.19(\operatorname{app~d}, J=16.5 \mathrm{~Hz}, 1 \mathrm{H}), 5.12(\mathrm{~d}, J=10.4 \mathrm{~Hz}, 1 \mathrm{H})$, $4.42(\operatorname{app~dt}, J=6.7,6.7 \mathrm{~Hz}, 2 \mathrm{H}), 2.54(\mathrm{q}, J=6.6 \mathrm{~Hz}, 2 \mathrm{H}) ;{ }^{13} \mathrm{C} \mathrm{NMR}\left(125 \mathrm{MHz}, \mathrm{CDCl}_{3}\right) \delta$ 196.1, $165.7,141.3,136.9,133.8,133.4,132.8,130.0,129.7,129.4,128.4,117.4,64.3,33.1$; HRMS $\left(\mathrm{Cl}^{+}\right) \mathrm{m} / \mathrm{z} 281.1172\left[(\mathrm{M}+\mathrm{H})^{+}\right.$; calc'd for $\left.\mathrm{C}_{18} \mathrm{H}_{17} \mathrm{O}_{3}: 281.1178\right]$.

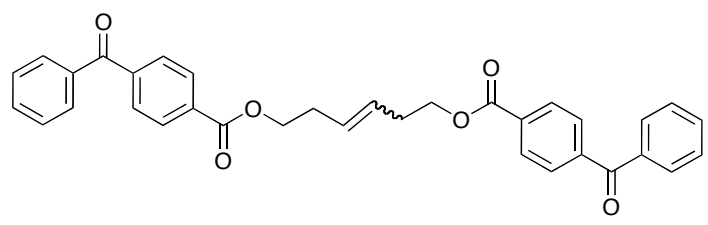

5

Ketone Dimer 5: To a solution of $\mathbf{S} 1(35 \mathrm{mg}, 0.125 \mathrm{mmol})$ in benzene $(1.3 \mathrm{~mL})$ was added Grubb's second generation catalyst $(10.0 \mathrm{mg}, 0.0117 \mathrm{mmol})$. This reddish solution was placed 
into a $60{ }^{\circ} \mathrm{C}$ bath and stirred for $3 \mathrm{~h}$. The reaction contents were then directly transferred to a preparatory $\mathrm{SiO}_{2}$ plate $(0.5 \mathrm{~mm}$, Merck) along with an additional $0.15 \mathrm{~mL}$ benzene rinse. The plate was developed with EtOAc/hexanes (1:7) and the product band was cut and rinsed with EtOAc $(25 \mathrm{~mL})$ to furnish dimer 5 as a colorless oil $(42 \mathrm{mg}, 1: 1$ ratio of presumed $E / Z$ isomers, $62 \%$ yield). Observed peak list for both inseparable olefin isomers: IR (film, $\mathrm{NaCl}$ ) 2953, 2908, 1722, 1659, 1444, 1272, $1103 \mathrm{~cm}^{-1} ;{ }^{1} \mathrm{H}$ NMR $\left(500 \mathrm{MHz}, \mathrm{CDCl}_{3}\right) \delta 8.02$ (app t, $\left.J=10.5 \mathrm{~Hz}, 4 \mathrm{H}\right)$, $7.81(\mathrm{~d}, J=7.7 \mathrm{~Hz}, 4 \mathrm{H}), 7.79(\mathrm{~d}, J=7.7 \mathrm{~Hz}, 4 \mathrm{H}), 7.60($ app t, $J=6.5 \mathrm{~Hz}, 2 \mathrm{H}), 7.48(\operatorname{app} \mathrm{t}, J=6.0$ $\mathrm{Hz}, 4 \mathrm{H}), 5.70-5.60(\mathrm{~m}, 2 \mathrm{H}), 4.38(\mathrm{q}, J=6.8 \mathrm{~Hz}, 4 \mathrm{H}), 2.61(\operatorname{app} \mathrm{q}, J=6.0 \mathrm{~Hz}, 2 \mathrm{H}), 2.53(\mathrm{q}, J=5.4$ $\mathrm{Hz}, 2 \mathrm{H}) ;{ }^{13} \mathrm{C}$ NMR $\left(125 \mathrm{MHz}, \mathrm{CDCl}_{3}\right) \delta 195.2,165.6,141.31,143.27$ (2), 136.9, 133.3, 132.8, 130.0, 129.7, 129.4, 128.5, 128.4 (2), 127.5, 64.6, 64.5 (2), 32.0, 27.0 (2); HRMS $\left(\mathrm{Cl}^{+}\right) \mathrm{m} / \mathrm{z}$ 533.1949 [(M+H) ${ }^{+}$; calc'd for $\mathrm{C}_{34} \mathrm{H}_{29} \mathrm{O}_{6}$ : 533.1964].

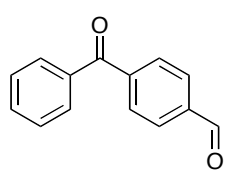

17

Aldehyde 17: To a suspension of 4-benzoylbenzoic acid $(1.24 \mathrm{~g}, 5.51 \mathrm{mmol})$ in THF $(12.0 \mathrm{~mL})$ at $-78{ }^{\circ} \mathrm{C}$ was added a solution of $\mathrm{BH}_{3} \cdot \mathrm{THF}(1.0 \mathrm{M}$ in THF, $18.0 \mathrm{~mL}, 18.0 \mathrm{mmol})$. The resulting solution was allowed to warm to $\mathrm{rt}$ and was stirred an additional $12 \mathrm{~h}$. The reaction was then poured into a saturated aqueous solution of $\mathrm{NH}_{4} \mathrm{Cl}(200 \mathrm{~mL})$ and the resulting emulsion was extracted with EtOAc $(3 \times 300 \mathrm{~mL})$. The combined organic extracts were further washed with water $(3 \times 100 \mathrm{~mL})$, dried $\left(\mathrm{Na}_{2} \mathrm{SO}_{4}\right)$, filtered and concentrated. The crude mixture of alcohols was subsequently dissolved in $\mathrm{CH}_{2} \mathrm{Cl}_{2}(26 \mathrm{~mL})$ and to this solution was added molecular sieves (4 $\AA$, $150 \mathrm{mg}$, powdered) and $\mathrm{N}$-methylmorpholine oxide (NMO, $1.30 \mathrm{~g}, 11.1 \mathrm{mmol}$ ). The resulting suspension was maintained at $\mathrm{rt}$ for $30 \mathrm{~min}$. At this time tetra- $n$-propylammonium perruthenate (TPAP, $73 \mathrm{mg}, 0.21 \mathrm{mmol}$ ) was added portionwise over $30 \mathrm{~min}$. to minimize any possible exothermic reaction process. After $1 \mathrm{~h}$ the reaction was concentrated to half volume with a rotary 
evaporator and the resulting dark suspension was directly subjected to $\mathrm{SiO}_{2}$ chromatography (EtOAc/hexanes $1: 1)$ to furnish the desired aldehyde 17 (800 mg, 69\% yield) as a white solid that was identical to the known literature values for this compound in all spectroscopic respects. For an assay of purity, the aldehyde $\mathbf{1 7}$ was subjected to combustion analysis: Anal. Calc'd for $\mathrm{C}_{14} \mathrm{H}_{10} \mathrm{O}_{2}: \mathrm{C}, 79.98 ; \mathrm{H}, 4.79$. Found: $\mathrm{C}, 79.61 ; \mathrm{H}, 4.70$.

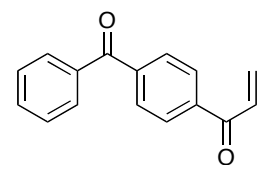

8

Enone 8: To a solution of aldehyde $17(35 \mathrm{mg}, 0.166 \mathrm{mmol})$ in $\mathrm{THF}(2.8 \mathrm{~mL})$ at $-78{ }^{\circ} \mathrm{C}$ was added an Aldrich solution of vinylmagnesium Grignard reagent (1.0 M THF, $0.15 \mathrm{~mL}, 0.15 \mathrm{mmol}$ ). After $1 \mathrm{~h}$ the reaction was quenched with $10 \mathrm{~mL}$ of saturated brine. The resulting emulsion was diluted with EtOAc $(100 \mathrm{~mL})$ and the bilayer was separated. The organic extract was further washed with water $(3 \times 30 \mathrm{~mL})$, dried $\left(\mathrm{Na}_{2} \mathrm{SO}_{4}\right)$, filtered and concentrated. The crude oil was purified by $\mathrm{SiO}_{2}$ column chromatography (3:1 hexanes/EtOAc) to furnish $33.6 \mathrm{mg}$ ( $85 \%$ yield) of an intermediate allylic alcohol. IR (film, NaCl) 3430, 1650, 1277, $923 \mathrm{~cm}^{-1} ;{ }^{1} \mathrm{H}$ NMR $(500 \mathrm{MHz}$, $\left.\mathrm{CDCl}_{3}\right) \delta$ 7.82-7.77 (m, 4H), $7.58(\operatorname{app~t}, J=7.5 \mathrm{~Hz}, 1 \mathrm{H}), 7.53-7.46(\mathrm{~m}, 4 \mathrm{H}), 6.04$ (ddt, $J=16.0$, 10.4, 6.3 Hz, 1H), $5.39(\operatorname{app~dt,~} J=16.0,1.4 \mathrm{~Hz}, 1 \mathrm{H}), 5.28(\mathrm{~d}, J=6.1 \mathrm{~Hz}, 1 \mathrm{H}), 5.23(\operatorname{app} \mathrm{dt}, J=$ 11.0, 1.1 Hz, 1H), 2.30-2.10 (br s, 1H); $\left.{ }^{13} \mathrm{C} \mathrm{NMR} \mathrm{(125} \mathrm{MHz,} \mathrm{CDCl}_{3}\right) \delta$ 196.3, 147.0, 139.7, 137.6, 138.6, 132.3, 130.3, 129.9, 128.2, 126.0, 115.9, 74.9; HRMS $\left(\mathrm{Cl}^{+}\right) \mathrm{m} / \mathrm{z} 239.1107\left[(\mathrm{M}+\mathrm{H})^{+}\right.$; calc'd for $\left.\mathrm{C}_{16} \mathrm{H}_{15} \mathrm{O}_{2}: 239.1072\right]$.

To a solution of the above alcohol in $\mathrm{CH}_{2} \mathrm{Cl}_{2}(1.0 \mathrm{~mL})$ was added $\mathrm{N}$-methylmorpholine oxide (NMO, $31 \mathrm{mg}, 0.26 \mathrm{mmol}$ ) and $0.1 \mathrm{~g}$ of $4 \AA$ molecular sieves. This suspension was stirred for 30 minutes at $\mathrm{rt}$ and next was added TPAP (tetra- $n$-propyl-ammonium perruthenate, $5 \mathrm{mg}, 0.010$ mmol). After $1 \mathrm{~h}$ the reaction was directly applied to $\mathrm{SiO}_{2}$ column chromatography $(9: 1$ hexanes/EtOAc) to furnish $20.0 \mathrm{mg}(70 \%$ yield) of the desired enone 8. IR (film, $\mathrm{NaCl})$ 1658, 
1651, 1600, 1410, 1230, $997 \mathrm{~cm}^{-1} ;{ }^{1} \mathrm{H}$ NMR $\left(500 \mathrm{MHz}, \mathrm{CDCl}_{3}\right) \delta 8.03$ (app dt, $J=8.5,1.5 \mathrm{~Hz}$ 2H), 7.88 (app dt, $J=6.5,1.5 \mathrm{~Hz}, 2 \mathrm{H}), 7.83-7.89(\mathrm{~m}, 2 \mathrm{H}), 7.61$ (app dt, $J=8.7,1.2 \mathrm{~Hz}, 1 \mathrm{H}), 7.50$ (app t, $J=8.7 \mathrm{~Hz}, 2 \mathrm{H}), 7.16(\mathrm{dd}, J=16.8,10.6 \mathrm{~Hz}, 1 \mathrm{H}), 6.46(\mathrm{dd}, J=16.8,1.4 \mathrm{~Hz}, 1 \mathrm{H}), 6.00$ (dd, $J=10.8,2.6 \mathrm{~Hz}, 1 \mathrm{H}) ;{ }^{13} \mathrm{C}$ NMR $\left(125 \mathrm{MHz}, \mathrm{CDCl}_{3}\right) \delta 195.8,190.6,141.1,139.9,136.9,132.9$, 132.3, 131.0, 130.02, 129.96, 128.5, 128.4; HRMS $\left(\mathrm{Cl}^{+}\right) \mathrm{m} / \mathrm{z} 237.0916\left[(\mathrm{M}+\mathrm{H})^{+}\right.$; calc'd for $\left.\mathrm{C}_{16} \mathrm{H}_{13} \mathrm{O}_{2}: 237.0927\right]$

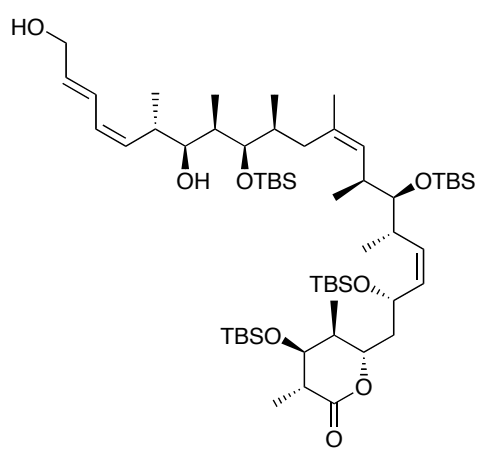

\section{$(+)-9$}

Diol (+)-9: To a resealable Schlenk tube was added trans-2-butene-1,4-diol ${ }^{1}$ (14.0 mg, 0.159 $\mathrm{mmol})$, dry benzene $(0.6 \mathrm{~mL})$ and Grubb's $2^{\text {nd }}$ generation catalyst $(4.0 \mathrm{mg}, 0.0047 \mathrm{mmol})$. This heterogeneous mixture was placed into a $45{ }^{\circ} \mathrm{C}$ bath and stirred for precisely 3.0 minutes. The Schlenk tube was then removed, cooled to rt and charged with a solution of diene (+)-3 $(7.0 \mathrm{mg}$, $0.0069 \mathrm{mmol}$ ) in $0.2 \mathrm{~mL}$ of benzene. The reaction was then reheated to $45^{\circ} \mathrm{C}$ for precisely 9.0 minutes and removed from the bath and placed in a solution of ice water to halt reaction. The reaction contents were then directly transferred to a preparatory $\mathrm{SiO}_{2}$ plate $(0.25 \mathrm{~mm}$, Merck) along with an additional $0.15 \mathrm{~mL}$ benzene rinse. The plate was developed with EtOAc/hexanes (1:9) with triplicate elution and the product band was cut and rinsed with EtOAc $(5 \mathrm{~mL})$ to furnish

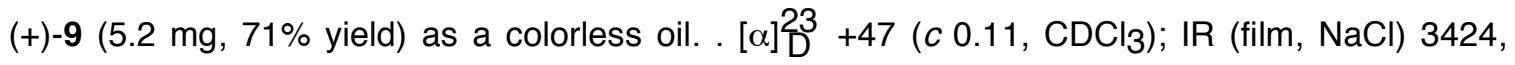
2957, 2930, 2928, 1718 (broad), 1654, 1462, 1379, 1360, 1252, 1098, 1046, 836, $775 \mathrm{~cm}^{-1} ;{ }^{1} \mathrm{H}$ NMR $\left(500 \mathrm{MHz}, \mathrm{CDCl}_{3}\right) \delta 6.54(\mathrm{dd}, J=15.3,11.1 \mathrm{~Hz}, 1 \mathrm{H}), 6.14($ app t, $J=11.1 \mathrm{~Hz}, 1 \mathrm{H}), 5.87$

\footnotetext{
${ }^{1}$ Prepared by the method disclosed in: Bauman, H.; Duthaler, R. O. Helv. Chem. Acta. 1988, 71, 1025.
} 
(app dt, $J=15.5,5.7 \mathrm{~Hz}, 1 \mathrm{H}), 5.35(\operatorname{app~t}, J=10.7 \mathrm{~Hz}, 1 \mathrm{H}), 5.31(\mathrm{~d}, J=8.1 \mathrm{~Hz}, 1 \mathrm{H}), 5.29(\mathrm{~d}, J=$ $7.8 \mathrm{~Hz}, 1 \mathrm{H}), 5.20(\operatorname{app~t}, J=10.3 \mathrm{~Hz}, 1 \mathrm{H}), 5.09(\mathrm{~d}, J=9.8 \mathrm{~Hz}, 1 \mathrm{H}), 4.79(\operatorname{app~t}, J=9.3 \mathrm{~Hz}, 1 \mathrm{H})$, $4.51(\operatorname{app} t, J=10.4 \mathrm{~Hz}, 1 \mathrm{H}), 4.22(\operatorname{app} d, J=5.7 \mathrm{~Hz}, 2 \mathrm{H}), 3.64-3.62(\mathrm{~m}, 2 \mathrm{H}), 3.35(\mathrm{dd}, J=7.7$, $3.0 \mathrm{~Hz}, 1 \mathrm{H}$ ), $3.26(\operatorname{app~t}, J=5.0 \mathrm{~Hz}, 1 \mathrm{H}), 2.80(\operatorname{app} \mathrm{br} q, J=8.4 \mathrm{~Hz}, 1 \mathrm{H}), 2.65-2.57(\mathrm{~m}, 2 \mathrm{H}), 2.55-$ $2.47(\mathrm{~m}, 1 \mathrm{H}), 2.17($ app t, $J=12.3 \mathrm{~Hz}, 1 \mathrm{H}), 1.90-1.70(\mathrm{~m}, 5 \mathrm{H}), 1.59(\mathrm{~s}, 3 \mathrm{H}), 1.22(\mathrm{~d}, J=7.4 \mathrm{~Hz}$ $3 \mathrm{H}), 0.97(\mathrm{~d}, J=6.5 \mathrm{~Hz}, 3 \mathrm{H}), 0.96(\mathrm{~d}, J=6.5 \mathrm{~Hz}, 3 \mathrm{H}), 0.91(\mathrm{~s}, 9 \mathrm{H}), 0.90(\mathrm{~s}, 9 \mathrm{H}), 0.89$ (d, $J=6.6$ $\mathrm{Hz}, 3 \mathrm{H}), 0.88(\mathrm{~s}, 9 \mathrm{H}), 0.88-0.86(\mathrm{~m}, 6 \mathrm{H}), 0.86(\mathrm{~s}, 9 \mathrm{H}), 0.74(\mathrm{~d}, J=6.6 \mathrm{~Hz}, 3 \mathrm{H}), 0.10(\mathrm{~s}, 6 \mathrm{H}), 0.07$ (s, 6H), $0.06(\mathrm{~m}, 6 \mathrm{H}), 0.051(\mathrm{~s}, 3 \mathrm{H}), 0.01(\mathrm{~s}, 3 \mathrm{H}) ;{ }^{13} \mathrm{C} \mathrm{NMR}\left(125 \mathrm{MHz}, \mathrm{CDCl}_{3}\right) \delta$ 173.3, 134.7, 133.5, 133.3, 132.4, 132.9, 131.4, 129.4, 126.3, 80.4, 78.8, 77.2, 76.6, 74.8, 64.6, 63.3, 44.1, $42.7,37.8,37.3,36.3,35.9,35.2,35.1,34.1,26.23,26.16,25.8,25.6,23.1,18.43,18.41,18.0$, $17.9,17.3,17.2,16.3,15.9,14.0,13.7,9.3,-3.0,-3.3,-3.7,-4.42,-4.46,-4.6,-4.92,-4.94 ;$ HRMS $\left(\mathrm{ES}^{+}\right) \mathrm{m} / \mathrm{z} 1259.7402\left[(\mathrm{M}+\mathrm{Na})^{+}\right.$; calc'd for $\left.\mathrm{C}_{57} \mathrm{H}_{112} \mathrm{O}_{8} \mathrm{NaSi}_{4}: 1259.7332\right]$.

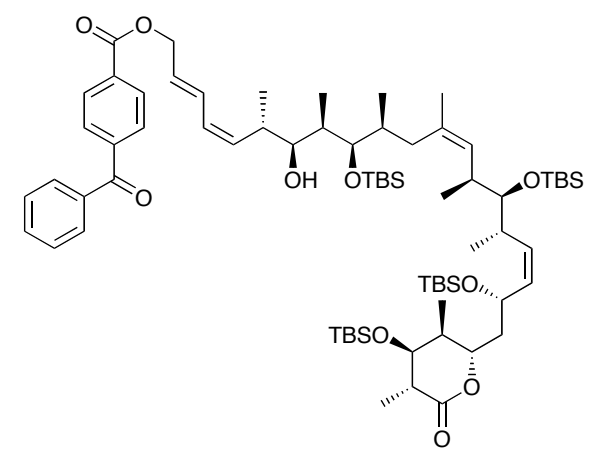

$(+)$-S2

Ester (+)-S2: To a resealable Schlenk tube was added diol (+)-9 (4.5 mg, $\left.4.3 \times 10^{-3} \mathrm{mmol}\right)$, $\mathrm{CH}_{2} \mathrm{Cl}_{2}(0.2 \mathrm{~mL})$, EDCI-Mel (8 mg, $\left.0.027 \mathrm{mmol}\right)$, HOBT (5.0 mg, $\left.0.037 \mathrm{mmol}\right)$, 4-benzoylbenzoic acid $(11.0 \mathrm{mg}, 0.049 \mathrm{mmol}$ ), and a single crystal of DMAP (approx. $1.0 \mathrm{mg}$, catalyst). The Schlenk tube was then sealed and the reaction was maintained at $\mathrm{rt}$ for $18 \mathrm{~h}$ until complete consumption of starting material was observed by TLC analysis. The reaction contents were then directly transferred to a preparatory $\mathrm{SiO}_{2}$ plate $(0.25 \mathrm{~mm}$, Merck) along with an additional 0.15 $\mathrm{mL} \mathrm{CH} \mathrm{Cl}_{2}$ rinse. The plate was developed with EtOAc/hexanes (1:9) and the product band was 
cut and rinsed with EtOAc (5 mL) to furnish (+)-S2 (5.2 mg, 97\% yield) as an amorphous solid. $[\alpha]_{\mathrm{D}}^{23}+21\left(c\right.$ 0.10, $\left.\mathrm{CDCl}_{3}\right) ; \mathrm{IR}$ (film, NaCl) 3452, 2954, 2919, 2858, 1724, 1718 (broad), 1663, 1462, 1375, 1257, 1095, 1042, 837, $771 \mathrm{~cm}^{-1} ;{ }^{1} \mathrm{H}$ NMR (500 MHz, CDCl 3 ) $\delta 8.16$ (app d, $J=8.0$ $\mathrm{Hz}, 2 \mathrm{H}), 7.84(\operatorname{app} d, J=8.0 \mathrm{~Hz}, 2 \mathrm{H}), 7.79(\operatorname{app} d, J=6.0 \mathrm{~Hz}, 2 \mathrm{H}), 7.61(\operatorname{app} t, J=6.3 \mathrm{~Hz}, 1 \mathrm{H})$, $7.46($ app t, $J=7.0 \mathrm{~Hz}, 1 \mathrm{H}), 6.69$ (dd, $J=14.5,11.5 \mathrm{~Hz}, 1 \mathrm{H}), 6.19(\operatorname{app} t, J=11.0 \mathrm{~Hz}, 1 \mathrm{H}), 5.92$ (app dt, $J=15.4,6.5 \mathrm{~Hz}, 1 \mathrm{H}), 5.41(\operatorname{app} t, J=11.4 \mathrm{~Hz}, 1 \mathrm{H}$ ), 5.29 (dd, $J=11.0,8.0 \mathrm{~Hz}, 1 \mathrm{H}$ ), 5.19 (app t, $J=10.3 \mathrm{~Hz}, 1 \mathrm{H}), 5.08(\mathrm{~d}, J=10.0 \mathrm{~Hz}, 1 \mathrm{H}), 4.91(\mathrm{~d}, J=6.3 \mathrm{~Hz}, 2 \mathrm{H}), 4.79(\operatorname{app~t}, J=9.4$ $\mathrm{Hz}, 1 \mathrm{H}), 4.51$ (app t, $J=10.7 \mathrm{~Hz}, 1 \mathrm{H}), 3.66-3.62(\mathrm{~m}, 2 \mathrm{H}), 3.35$ (app dt, $J=12.0,2.5 \mathrm{~Hz}, 1 \mathrm{H}), 3.26$ (app t, $J=4.7 \mathrm{~Hz}, 1 \mathrm{H}), 2.82(\mathrm{br} q, J=7.3 \mathrm{~Hz}, 1 \mathrm{H}), 2.64-2.58(\mathrm{~m}, 2 \mathrm{H}), 2.52-2.45(\mathrm{~m}, 2 \mathrm{H}), 2.19$ (app t, $J=11.5 \mathrm{~Hz}, 1 \mathrm{H}), 1.92-1.68(\mathrm{~m}, 6 \mathrm{H}), 1.55(\mathrm{~s}, 3 \mathrm{H}), 1.22$ (d, $J=7.6 \mathrm{~Hz}, 3 \mathrm{H}), 0.97$ (d, $J=7.0$ $\mathrm{Hz}, 3 \mathrm{H}$ ), 0.95 (d, J=7.0 Hz, 3H), 0.94 (d, J=7.0 Hz, 3H), 0.92 (s, 9H), 0.90 (s, 9H), 0.92-0.89 (s, 9H), $0.87(\mathrm{~s}, 9 \mathrm{H}), 0.83(\mathrm{~m}, 6 \mathrm{H}), 0.74(\mathrm{~d}, J=6.8 \mathrm{~Hz}, 3 \mathrm{H}), 0.10(\mathrm{~s}, 3 \mathrm{H}), 0.09(\mathrm{~s}, 3 \mathrm{H}), 0.07(\mathrm{~s}, 6 \mathrm{H})$, 0.06 (s, 6H), 0.05 (s, 3H), 0.01 (s, 3H); ${ }^{13} \mathrm{C}$ NMR (125 MHz, CDCl3) $\delta$ 195.9, 173.3, 165.5, 141.3, $136.9,136.3,133.5,133.3,132.8,132.4,132.0,131.3,129.6,129.7,129.8,129.4,129.0,128.4$ 127.3, 80.4, 78.9, 77.2, 76.4, 74.8, 65.5, 64.5, 44.1, 42.7, 37.7, 37.3, 36.5, 36.0, 35.2, 35.1, 34.2, 26.3, 26.2, 25.8, 25.7, 23.2, 18.37, 18.21, 18.0, 17.9, 17.2, 17.1, 16.3, 16.0, 14.0, 13.5, 9.3, -3.0, 3.4, -3.8, -4.41,-4.44, -4.6, -4.92, -4.94; HRMS $\left(\mathrm{ES}^{+}\right) \mathrm{m} / \mathrm{z} 1267.7878\left[(\mathrm{M}+\mathrm{Na})^{+}\right.$; calc'd for $\left.\mathrm{C}_{71} \mathrm{H}_{120} \mathrm{O}_{10} \mathrm{NaSi}_{4}: 1267.7856\right]$.

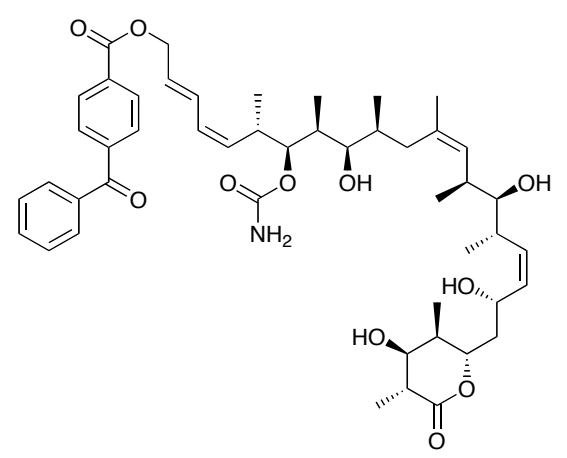

$(+)-10$ 
Ester (+)-10: A solution of alcohol (+)-S2 $\left(4.6 \mathrm{mg}, 3.7 \times 10^{-3} \mathrm{mmol}\right)$ in $\mathrm{CH}_{2} \mathrm{Cl}_{2}(0.8 \mathrm{~mL})$ was treated with $\mathrm{Cl}_{3} \mathrm{CCON}=\mathrm{C}=\mathrm{O}(10 \mu \mathrm{L}, 1.0 \mathrm{M}$ benzene solution, $0.010 \mathrm{mmol})$ at $\mathrm{rt}$ and stirred for 30 min. The reaction mixture was loaded directly onto neutral $\mathrm{Al}_{2} \mathrm{O}_{3}(1.0 \times 4.0 \mathrm{~cm}$ plug $)$. After $4 \mathrm{~h}$, the material was flushed from the $\mathrm{Al}_{2} \mathrm{O}_{3}(\mathrm{EtOAc} / \mathrm{MeOH}, 15: 1,20 \mathrm{~mL})$, concentrated, and diluted with $0.8 \mathrm{~mL} \mathrm{MeOH}$. To this solution was added aqueous hydrochloric acid $(3 \mathrm{~N}, 1.0 \mathrm{~mL})$ in 0.05 $\mathrm{mL}$ portions over 4 hours at a rate which minimized precipitation (ca. 10 to 15 min intervals). An additional $0.5 \mathrm{~mL}$ of $3 \mathrm{~N}$ aq HCl was added over $1 \mathrm{~h}$ at $15 \mathrm{~min}$ intervals, and the sides of the flask/stir bar were rinsed with $1 \mathrm{~mL}$ of $\mathrm{MeOH}$. After $12 \mathrm{~h}$ total time, an additional $0.2 \mathrm{~mL}$ of $3 \mathrm{~N}$ aq $\mathrm{HCl}$ was added in one portion, and the resulting solution was stirred for an additional $2 \mathrm{~h}$ at rt. At this time the reaction was diluted with $35 \mathrm{~mL}$ of EtOAc and the reaction mixture was slowly quenched with solid $\mathrm{NaHCO}_{3}(80 \mathrm{mg})$ until cessation of bubbling. The solution was then filtered through celite and washed with additional EtOAc $(5.0 \mathrm{~mL})$. The organic layer was dried ( $\left.\mathrm{Na}_{2} \mathrm{SO}_{4}\right)$, filtered, and concentrated. Residual water was removed by co-evaporation with EtOH $(3 \times 3 \mathrm{~mL})$. Subjection of the crude residue to $\mathrm{SiO}_{2}$ chromatography furnished $(+)-10(2.0 \mathrm{mg}$, $65 \%$ yield) as an amorphous solid. $[\alpha]_{\mathrm{D}}^{23}+32\left(c 0.10, \mathrm{CD}_{3} \mathrm{CN}\right.$; IR (film, $\left.\mathrm{NaCl}\right) 3426,2978,2928$, 2878, 1721, 1710 (broad), 1658, 1600, 1445, 1386, 1272, 1102, 1044, 991, $922 \mathrm{~cm}^{-1} ;{ }^{1} \mathrm{H}$ NMR (500 MHz, CD $3 \mathrm{CN}$ ) $\delta 8.16(\operatorname{app~d}, J=8.0 \mathrm{~Hz}, 2 \mathrm{H}), 7.87$ (app d, $J=8.2 \mathrm{~Hz}, 2 \mathrm{H}), 7.81$ (app d, $J=$ $7.5 \mathrm{~Hz}, 2 \mathrm{H}), 7.69(\operatorname{appt}, J=7.5 \mathrm{~Hz}, 1 \mathrm{H}), 7.46(\operatorname{app~t}, J=7.8 \mathrm{~Hz}, 1 \mathrm{H}), 6.76(\mathrm{dd}, J=14.5,11.8 \mathrm{~Hz}$, 1H), $6.14(\operatorname{app~t}, J=11.1 \mathrm{~Hz}, 1 \mathrm{H}), 5.92(\operatorname{app~dt}, J=15.0,6.0 \mathrm{~Hz}, 1 \mathrm{H}), 5.57($ app t, $J=10.2 \mathrm{~Hz}$, 1H), $5.49(\operatorname{app~t}, J=11.5 \mathrm{~Hz}, 1 \mathrm{H}), 5.40(\mathrm{dd}, J=11.0,8.7 \mathrm{~Hz}, 1 \mathrm{H}), 5.12-5.00(\mathrm{br} \mathrm{s}, 2 \mathrm{H}), 4.98(\mathrm{~d}, J$ $=10.0 \mathrm{~Hz}, 1 \mathrm{H}), 4.94(\mathrm{dd}, J=10.0,6.3 \mathrm{~Hz}, 1 \mathrm{H}), 4.74(\mathrm{dd}, J=7.8,4.5 \mathrm{~Hz}, 1 \mathrm{H}), 4.50-4.42(\mathrm{~m}, 2 \mathrm{H})$, $3.63(\operatorname{app~q,~} J=4.5 \mathrm{~Hz}, 1 \mathrm{H}), 3.29(\mathrm{~d}, J=4.4 \mathrm{~Hz}, 1 \mathrm{H}), 3.19-3.14(\mathrm{~m}, 1 \mathrm{H}), 3.14-3.06(\mathrm{~m}, 2 \mathrm{H}), 2.77$ (d, $J=5.6 \mathrm{~Hz}, 1 \mathrm{H}), 2.68(\mathrm{~d}, J=6.0 \mathrm{~Hz}, 1 \mathrm{H}), 2.65-2.54(\mathrm{~m}, 4 \mathrm{H}), 2.60(\mathrm{~d}, J=6.3 \mathrm{~Hz}, 1 \mathrm{H}), 2.27$ (app q, $J=6.4 \mathrm{~Hz}, 1 \mathrm{H}), 1.80-1.59(\mathrm{~m}, 6 \mathrm{H}), 1.57(\mathrm{~s}, 3 \mathrm{H}), 1.49($ app t, $J=13.0 \mathrm{~Hz}, 1 \mathrm{H}), 1.20(\mathrm{~d}, J=7.3$ $\mathrm{Hz}, 3 \mathrm{H}), 1.02(\mathrm{~d}, J=7.0 \mathrm{~Hz}, 3 \mathrm{H}), 0.99(\mathrm{~d}, J=7.1 \mathrm{~Hz}, 3 \mathrm{H}), 0.97(\mathrm{~d}, J=6.8 \mathrm{~Hz}, 3 \mathrm{H}), 0.87(\mathrm{~d}, J=$ $6.5 \mathrm{~Hz}, 3 \mathrm{H}), 0.81(\mathrm{~d}, J=6.8 \mathrm{~Hz}, 3 \mathrm{H}), 0.73(\mathrm{~d}, J=6.1 \mathrm{~Hz}, 3 \mathrm{H}) ;{ }^{13} \mathrm{C} \mathrm{NMR}\left(125 \mathrm{MHz}, \mathrm{CD}_{3} \mathrm{CN}\right) \delta$ 
$195.6,184.1,173.5,157.3,145.4,141.4,138.2,134.2,132.9,132.85,132.78,132.73,130.1$, $129.8,129.7,129.3,128.9,128.5,127.8,127.4,78.7,78.1,76.5,76.1,75.1,72.2,70.1,43.1$, $41.2,37.5,36.2,35.8,35.6,35.3,33.7,33.2,22.3,19.5,18.7,17.1,16.6,14.7,12.0,8.1$; HRMS $\left(\mathrm{ES}^{+}\right) \mathrm{m} / \mathrm{z} 832.4600\left[(\mathrm{M}+\mathrm{H})^{+}\right.$; calc'd for $\left.\mathrm{C}_{48} \mathrm{H}_{66} \mathrm{NO}_{11}: 832.4636\right]$.

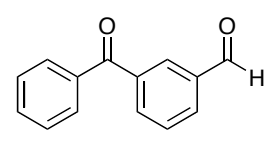

16

Aldehyde 16: To a suspension of 3-benzoylbenzoic acid $(1.24 \mathrm{~g}, 5.51 \mathrm{mmol})$ in THF $(12.0 \mathrm{~mL})$ at $-78{ }^{\circ} \mathrm{C}$ was added a THF solution of $\mathrm{BH}_{3} \cdot \mathrm{THF}(1.0 \mathrm{M}$ in THF, $18.0 \mathrm{~mL}, 18.0 \mathrm{mmol})$. The resulting solution was allowed to warm to rt and was stirred for an additional $12 \mathrm{~h}$. The reaction was then poured into a saturated aqueous solution of $\mathrm{NH}_{4} \mathrm{Cl}(200 \mathrm{~mL})$ and the resulting emulsion was extracted with EtOAc $(3 \times 300 \mathrm{~mL})$. The combined organic extracts were further washed with water $(3 \times 100 \mathrm{~mL})$, dried ( $\left.\mathrm{Na}_{2} \mathrm{SO}_{4}\right)$, filtered and concentrated. The crude mixture of alcohols was subsequently dissolved in $\mathrm{CH}_{2} \mathrm{Cl}_{2}(26 \mathrm{~mL})$ and to this solution was added molecular sieves (4 $\AA$, $150 \mathrm{mg}$, powdered) and $\mathrm{N}$-methylmorpholine oxide (NMO, $1.30 \mathrm{~g}, 11.1 \mathrm{mmol})$. The resulting suspension was maintained at $\mathrm{rt}$ for $30 \mathrm{~min}$. At this time tetra- $n$-propylammonium perruthenate (TPAP, $73 \mathrm{mg}, 0.21 \mathrm{mmol}$ ) was added portionwise over $30 \mathrm{~min}$. to minimize any possible exothermic reaction process. After $1 \mathrm{~h}$ the reaction was concentrated to half volume with a rotary evaporator and the resulting dark suspension was directly subjected to $\mathrm{SiO}_{2}$ chromatography (EtOAc/hexanes $1: 1)$ to furnish 16 (800 $\mathrm{mg}, 69 \%$ yield) as a white solid that was identical to the known literature values for this compound in all spectroscopic respects. $\mathrm{Mp}=62-63^{\circ} \mathrm{C}$, lit. value $\left(61-63^{\circ} \mathrm{C}\right)$. For an assay of purity, aldehyde 16 was subjected to combustion analysis: Anal. Calc'd for $\mathrm{C}_{14} \mathrm{H}_{10} \mathrm{O}_{2}: \mathrm{C}, 79.98 ; \mathrm{H}, 4.79$. Found: $\mathrm{C}, 79.61 ; \mathrm{H}, 4.70$.

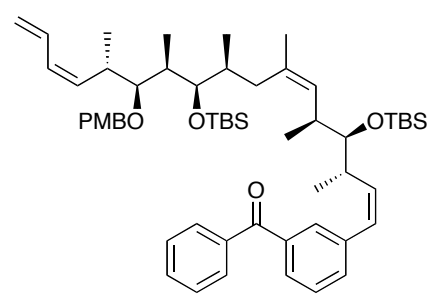


$(+)-18$

Tetraene (+)-18: Procedure For Solid Template Wittig Reaction: To a suspension of ZnO (15.0 $\mathrm{mg}, 0.19 \mathrm{mmol}), \mathrm{Cs}_{2} \mathrm{CO}_{3}(15.0 \mathrm{mg}, 0.050 \mathrm{mmol}), \mathrm{K}_{2} \mathrm{CO}_{3}(2.0 \mathrm{mg}, 0.014 \mathrm{mmol})$, and aldehyde $16(50.0 \mathrm{mg}, 0.238 \mathrm{mmol})$ in 1,4 dioxane $(0.2 \mathrm{~mL})$ was added a solution of phosphonium salt $(+)-$ 15 (23.0 mg, $0.021 \mathrm{mmol}, 8 / 1 \mathrm{Z} / \mathrm{E}$ mixture of diene isomers) in 1,4 dioxane $(0.4 \mathrm{~mL})$. The reaction vessel was heated to $48{ }^{\circ} \mathrm{C}$ in a resealable tube and maintained for $3 \mathrm{~h}$. The suspension was allowed to cool to rt, settle for $1 \mathrm{~h}$, and then the suspension was filtered through a plug of celite $(0.5 \times 3 \mathrm{~cm})$. The resulting plug was rinsed with EtOAc $(4 \mathrm{ml})$. This resulting solution was concentrated to one quarter volume and applied directly to preparatory TLC $\mathrm{SiO}_{2}$ chromatography (Merck, $0.5 \mathrm{~mm}, 1: 10 \mathrm{EtOAc/hexanes).} \mathrm{The} \mathrm{resulting} \mathrm{product} \mathrm{band} \mathrm{was} \mathrm{cut} \mathrm{and}$ the $\mathrm{SiO}_{2}$ rinsed with EtOAc $(20 \mathrm{~mL})$ to furnish Wittig product $(+)-\mathbf{1 8}(15.1 \mathrm{mg}, \mathbf{8 0} \%$ yield $)$ as a foam.

Alternative procedure: To a $-30^{\circ} \mathrm{C}$ solution of previously dried phosphonium salt $(+)-15(12 \mathrm{mg}$, $0.011 \mathrm{mmol}, 12: 1$ ratio of $Z / E$ diene isomers $)$ in THF $(0.7 \mathrm{~mL})$ was added a solution of NaHMDS (1.0 $\mathrm{M}$ in THF, $0.015 \mathrm{~mL}, 0.015 \mathrm{mmol}$ ) to furnish an intermediate ylide that was warmed to $-10^{\circ} \mathrm{C}$ for $10 \mathrm{~min}$ and recooled to $-20^{\circ} \mathrm{C}$ at which point a solution of aldehyde $16(15.0 \mathrm{mg}, 0.070 \mathrm{mmol})$ in THF $(0.40 \mathrm{~mL})$ was added. The reaction was maintained for $30 \mathrm{~min}$. at $-20^{\circ} \mathrm{C}$, allowed to warm to $0{ }^{\circ} \mathrm{C}$, and was then maintained for $1 \mathrm{~h}$ followed by the addition of a saturated solution of $\mathrm{NH}_{4} \mathrm{Cl}$ $(1.0 \mathrm{~mL})$. The emulsion was extracted with EtOAc $(3 \times 5 \mathrm{~mL})$ and the combined organics were dried $\left(\mathrm{Na}_{2} \mathrm{SO}_{4}\right)$, filtered and concentrated. Subjection of the crude residue to dried $\mathrm{SiO}_{2}$ chromatography (hexanes/EtOAc, 9:1) furnished (+)-18 (4.1 mg, 42\% yield). $[\alpha]^{23}+57(c 1.03$, $\mathrm{CH}_{2} \mathrm{Cl}_{2}$ ); IR (film, NaCl) 2957, 2930, 2847, 1661, 1610, 1512, 1459, 1250, 1086, 1040, 834, 772 $\mathrm{cm}^{-1} ;{ }^{1} \mathrm{H}$ NMR $\left(500 \mathrm{MHz}, \mathrm{CDCl}_{3}\right) \delta 7.78(\operatorname{app~d}, J=8.0 \mathrm{~Hz}, 2 \mathrm{H}), 7.69$ (br s, $\left.1 \mathrm{H}\right), 7.62(\mathrm{dd}, J=7.0$, $1.3 \mathrm{~Hz}, 1 \mathrm{H}), 7.57$ (app t, $J=7.4 \mathrm{~Hz}, 1 \mathrm{H}), 7.52-7.50(\mathrm{~m}, 3 \mathrm{H}), 7.38-7.30(\mathrm{~m}, 1 \mathrm{H}), 7.28(\mathrm{~d}, J=7.5$ $\mathrm{Hz}, 2 \mathrm{H}), 6.85$ (d, $J=7.5 \mathrm{~Hz}, 2 \mathrm{H}), 6.56$ (ddd, $J=16.1,10.7,10.6 \mathrm{~Hz}, 1 \mathrm{H}), 6.38(\mathrm{~d}, J=12.0 \mathrm{~Hz}$, 1H), $6.05($ app t, $J=11.1 \mathrm{~Hz}, 1 \mathrm{H}), 5.79(\operatorname{app~t}, J=10.5 \mathrm{~Hz}, 1 \mathrm{H}), 5.59($ app t, $J=10.4 \mathrm{~Hz}, 1 \mathrm{H})$, 
$5.20(\mathrm{~d}, J=16.0 \mathrm{~Hz}, 1 \mathrm{H}), 5.06(\mathrm{~d}, J=11.0 \mathrm{~Hz}, 1 \mathrm{H}), 4.89(\mathrm{~d}, J=10.1 \mathrm{~Hz}, 1 \mathrm{H}), 4.51\left(\mathrm{ABq}, J_{\mathrm{AB}}=\right.$ 10.6 Hz, vAB = 41.56 Hz, 2H), $3.79(\mathrm{~s}, 3 \mathrm{H}), 3.40($ app t, $J=2.4 \mathrm{~Hz}, 1 \mathrm{H}), 3.28(\mathrm{dd}, J=7.0,3.6 \mathrm{~Hz}$, 1H), $3.23(\mathrm{dd}, J=7.5,3.5 \mathrm{~Hz}, 1 \mathrm{H}), 3.04-2.91(\mathrm{~m}, 2 \mathrm{H}), 2.37$ (app dt, $J=10.0,6.8 \mathrm{~Hz}, 1 \mathrm{H}), 1.92$ (app t, $J=11.8 \mathrm{~Hz}, 1 \mathrm{H}), 1.80-1.72(\mathrm{~m}, 1 \mathrm{H}), 1.69-1.61(\mathrm{~m}, 1 \mathrm{H}), 1.38-1.30(\mathrm{~m}, 1 \mathrm{H}), 1.34(\mathrm{~s}, 3 \mathrm{H})$, $1.13(\mathrm{~d}, J=7.1 \mathrm{~Hz}, 3 \mathrm{H}), 0.99(\mathrm{~d}, J=7.0 \mathrm{~Hz}, 3 \mathrm{H}), 0.95(\mathrm{~d}, J=6.8 \mathrm{~Hz}, 3 \mathrm{H}), 0.93(\mathrm{~s}, 9 \mathrm{H}), 0.91$ (s, $9 \mathrm{H}), 0.84(\mathrm{~d}, J=7.0 \mathrm{~Hz}, 3 \mathrm{H}), 0.65(\mathrm{~d}, J=6.5 \mathrm{~Hz}, 3 \mathrm{H}), 0.10(\mathrm{~s}, 3 \mathrm{H}), 0.09(\mathrm{~s}, 3 \mathrm{H}), 0.03(\mathrm{~s}, 3 \mathrm{H})$, 0.02 (s, 3H); ${ }^{13} \mathrm{C}$ NMR $\left(125 \mathrm{MHz}, \mathrm{CDCl}_{3}\right) \delta 196.3,159.0,137.9,137.7,137.4,136.3,134.4$ $132.9,132.5,135.2,131.1,129.9,129.7,129.1,129.0,129.00,128.3,128.1,128.0,127.9,127.4$, $117.5,113.6,84.6,80.9,76.7,75.0,68.1,55.2,40.0,36.8,36.6,35.6,35.3,35.2,26.23,26.22$, 22.6, 18.6, 18.5, 18.4, 17.5, 14.8, 10.4, -3.2, -3.3, -3.4, -3.7; HRMS (ES $\left.{ }^{+}\right) \mathrm{m} / z$ 915.5767 $\left[(\mathrm{M}+\mathrm{Na})^{+}\right.$; calc'd for $\left.\mathrm{C}_{56} \mathrm{H}_{84} \mathrm{NaO}_{5} \mathrm{Si}_{2}: 915.5755\right]$

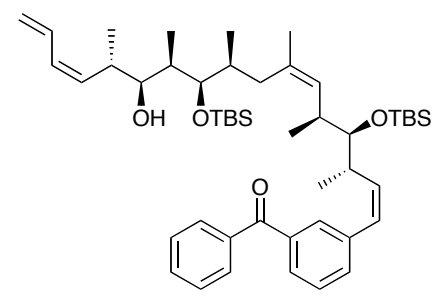

(+)-S3

Alcohol (+)-S3: An emulsion of PMB ether $(+)-18(17.9 \mathrm{mg}, 0.020 \mathrm{mmol})$ in $\mathrm{CH}_{2} \mathrm{Cl}_{2}(2.8 \mathrm{~mL})$ and of $\mathrm{pH} 7$ buffer $(0.5 \mathrm{~mL})$ was treated with DDQ $(16.0 \mathrm{mg}, 0.050 \mathrm{mmol})$ at $0{ }^{\circ} \mathrm{C}$. After $2.5 \mathrm{~h}$ the reaction mixture was quenched with $\mathrm{NaHCO}_{3}$ saturated solution $(1.0 \mathrm{~mL})$ and extracted with EtOAc $(3 \times 25 \mathrm{~mL})$. The combined organics were washed with additional $\mathrm{NaHCO}_{3}$ saturated solution $(10 \mathrm{~mL})$, dried $\left(\mathrm{Na}_{2} \mathrm{SO}_{4}\right)$, filtered and concentrated. The residue was subjected to $\mathrm{SiO}_{2}$ chromatography $\left(9: 1\right.$ hexanes/EtOAc) to furnish alcohol $(+)-\mathbf{S 3}(12.4 \mathrm{mg}, 80 \%$ yield $) .[\alpha]_{\mathrm{D}}^{23}+44$ (c 0.95, $\mathrm{CH}_{2} \mathrm{Cl}_{2}$ ); IR (film, NaCl) 3528, 2957, 2921, 2858, 1660, 1591, 1460, 1379, 1275, 1252 , 1084, 831, $772 \mathrm{~cm}^{-1} ;{ }^{1} \mathrm{H}$ NMR (500 MHz, $\mathrm{CDCl}_{3}$ ) $\delta 7.79$ (app d, J=6.9 Hz, 2H), 7.69 (br s, $\left.1 \mathrm{H}\right)$, $7.61(\operatorname{app~d}, J=6.5 \mathrm{~Hz}, 1 \mathrm{H}), 7.58(\operatorname{app~t}, J=7.5 \mathrm{~Hz}, 1 \mathrm{H}), 7.49(\mathrm{~d}, J=6.5 \mathrm{~Hz}, 1 \mathrm{H}), 7.47(\operatorname{app~t}, J=$ $7.8 \mathrm{~Hz}, 2 \mathrm{H}), 7.37(\mathrm{~d}, J=7.7 \mathrm{~Hz}, 1 \mathrm{H}), 6.62(\mathrm{ddd}, J=15.6,10.6,10.6 \mathrm{~Hz}, 1 \mathrm{H}), 6.38(\mathrm{~d}, J=11.9 \mathrm{~Hz}$, 
1H), $6.15($ app t, $J=11.1 \mathrm{~Hz}, 1 \mathrm{H}), 5.82(\operatorname{app~t}, J=10.8 \mathrm{~Hz}, 1 \mathrm{H}), 5.38(\operatorname{app~t}, J=10.5 \mathrm{~Hz}, 1 \mathrm{H})$, $5.20(\mathrm{dd}, J=16.0,1.5 \mathrm{~Hz}, 1 \mathrm{H}), 5.14(\mathrm{~d}, J=10.2 \mathrm{~Hz}, 1 \mathrm{H}), 4.89(\mathrm{~d}, J=10.1 \mathrm{~Hz}, 1 \mathrm{H}), 3.54(\mathrm{dd}, J=$ 5.4, 3.8 Hz, 1H), 3.34-3.28 (m, 2H), 3.02 (ddd, $J=10.5,7.5,3.0 \mathrm{~Hz}, 1 \mathrm{H}), 2.81$ (app dt, $J=12.5$, 7.0 Hz, 1H), 2.41 (app dt, $J=12.5,7.0 \mathrm{~Hz}, 1 \mathrm{H}), 2.05$ (app t, $J=12.5 \mathrm{~Hz}, 1 \mathrm{H}), 1.76-1.67(\mathrm{~m}, 3 \mathrm{H})$, $1.41(\mathrm{br} \mathrm{d}, J=13.5 \mathrm{~Hz}, 1 \mathrm{H}), 1.33(\mathrm{~s}, 3 \mathrm{H}), 1.01(\mathrm{~d}, J=7.0 \mathrm{~Hz}, 3 \mathrm{H}), 0.98(\mathrm{~d}, J=6.8 \mathrm{~Hz}, 3 \mathrm{H}), 0.92$ (s, 9H), $0.91(\mathrm{~d}, J=6.5 \mathrm{~Hz}, 3 \mathrm{H}), 0.90(\mathrm{~s}, 9 \mathrm{H}), 0.87(\mathrm{~d}, J=6.7 \mathrm{~Hz}, 3 \mathrm{H}), 0.67(\mathrm{~d}, J=6.7 \mathrm{~Hz}, 3 \mathrm{H})$,

0.08 (s, 6H), 0.04 (s, 3H), 0.03 (s, 3H); $\left.{ }^{13} \mathrm{C} \mathrm{NMR} \mathrm{(125} \mathrm{MHz,} \mathrm{CDCl}_{3}\right) \delta$ 196.3, 138.0, 137.6, 137.3, $136.2,134.6,132.7,132.4,132.3,132.1,130.7,130.4,130.1,130.0,128.2,128.0,127.9,127.4$, $118.3,81.0,78.5,76.2,38.3,37.0,36.5,36.1,35.9,34.5,26.21,26.19,22.7,18.8,18.5,18.4$, 17.6, 17.3, 13.7, 9.3, -3.2, -3.4, -3.5, -3.7; HRMS $\left(\mathrm{ES}^{+}\right) \mathrm{m} / \mathrm{z} 795.5123\left[(\mathrm{M}+\mathrm{Na})^{+}\right.$; calc'd for $\left.\mathrm{C}_{48} \mathrm{H}_{76} \mathrm{NaO}_{4} \mathrm{Si}_{2}: 795.5180\right]$.

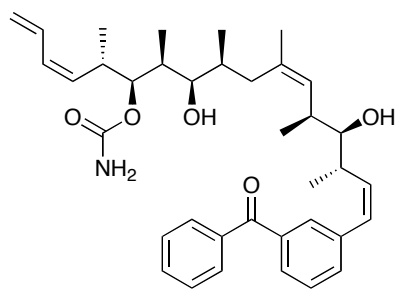

$(+)-20$

Carbamate (+)-20: A solution of alcohol (+)-S3 (11.0 mg, $0.0142 \mathrm{mmol}$ ) was treated with $\mathrm{Cl}_{3} \mathrm{CCON}=\mathrm{C}=\mathrm{O}(1.0 \mathrm{M}$ benzene, $0.21 \mathrm{~mL}, 0.21 \mathrm{mmol})$ and stirred for 30 minutes before direct loading onto neutral $\mathrm{Al}_{2} \mathrm{O}_{3}(1.0 \times 4.0 \mathrm{~cm}$ plug $)$. After $4 \mathrm{~h}$, the material was flushed from the $\mathrm{Al}_{2} \mathrm{O}_{3}$ (EtOAc/MeOH, 15:1, $20 \mathrm{~mL}$ ), concentrated and subsequently diluted with $0.8 \mathrm{~mL} \mathrm{MeOH}$. To this resulting solution was added aqueous hydrochloric acid $(3 \mathrm{~N}, 1.0 \mathrm{~mL})$ in $0.05 \mathrm{~mL}$ portions over 4 hours at a rate which minimized precipitation (ca. 10 to 15 min intervals). An additional $0.1 \mathrm{~mL}$ of $3 \mathrm{~N}$ aq HCl was added over $2 \mathrm{~h}$ at 15 min intervals, and the sides of the flask/stir bar were rinsed with $1.5 \mathrm{~mL}$ of $\mathrm{MeOH}$. After $14 \mathrm{~h}$ total time, an additional $0.3 \mathrm{~mL}$ of $3 \mathrm{~N}$ aq HCl was added in one portion, and the resulting solution was stirred for an additional $2 \mathrm{~h}$ at $\mathrm{rt}$. At this time the reaction was diluted with $15 \mathrm{~mL}$ of EtOAc and the reaction mixture was slowly quenched with solid 
$\mathrm{NaHCO}_{3}(80 \mathrm{mg})$ until cessation of bubbling. The solution was then filtered through celite and rinsed with an additional $10.0 \mathrm{~mL}$ of EtOAc. The organic was dried $\left(\mathrm{Na}_{2} \mathrm{SO}_{4}\right)$, filtered and concentrated. The resulting residue was subjected to $\mathrm{SiO}_{2}$ chromatography $\left(95: 5 \mathrm{CH}_{2} \mathrm{Cl}_{2} / \mathrm{MeOH}\right)$ to provide (+)-20 (5.5 mg, 66\% yield). $[\alpha]_{\mathrm{D}}^{23}+41\left(c \quad 0.35, \mathrm{C}_{6} \mathrm{H}_{6}\right)$; IR (film, $\left.\mathrm{NaCl}\right) 3402,2962$, 2824, 2860, 1714 (broad), 1654, 1596, 1452, 1389, 1319, 1278, 1045, $990 \mathrm{~cm}^{-1} ;{ }^{1} \mathrm{H}$ NMR (500 $\left.\mathrm{MHz}, \mathrm{C}_{6} \mathrm{D}_{6}\right) \delta 7.95(\mathrm{~s}, 1 \mathrm{H}), 7.77(\mathrm{~d}, J=7.0 \mathrm{~Hz}, 2 \mathrm{H}), 7.50(\mathrm{~d}, J=7.0 \mathrm{~Hz}, 1 \mathrm{H}), 7.47(\mathrm{~d}, J=7.0 \mathrm{~Hz}$ 1H), 7.14-7.10 (m, 2H), $7.06(\operatorname{app~q,~J=8.2~Hz,~2H),~} 6.57$ (ddd, $J=17.0,10.6,10.6 \mathrm{~Hz}, 1 \mathrm{H}), 6.38$ (d, $J=12.0 \mathrm{~Hz}, 1 \mathrm{H}), 6.06(\operatorname{app~t}, J=11.0 \mathrm{~Hz}, 1 \mathrm{H}$ ), 5.87 (app t, $J=11.2 \mathrm{~Hz}, 1 \mathrm{H}$ ), 5.50 (app t, $J=$ $10.5 \mathrm{~Hz}, 1 \mathrm{H}), 5.15(\mathrm{~d}, J=16.7 \mathrm{~Hz}, 1 \mathrm{H}), 5.05-4.99(\mathrm{~m}, 2 \mathrm{H}), 4.92(\mathrm{~d}, J=10.1 \mathrm{~Hz}, 1 \mathrm{H}), 4.01-3.92$ (br s, 2H), $3.22($ app t, $J=5.0 \mathrm{~Hz}, 1 \mathrm{H}), 3.19-3.09(\mathrm{~m}, 1 \mathrm{H}), 3.05-3.00(\mathrm{~m}, 2 \mathrm{H}) 2.50($ app q, $J=7.5$ $\mathrm{Hz}, 1 \mathrm{H}), 2.06-1.95(\mathrm{~m}, 3 \mathrm{H}), 1.56(\mathrm{~s}, 3 \mathrm{H}), 1.45(\operatorname{app~d}, J=8.5 \mathrm{~Hz}, 1 \mathrm{H}), 1.40-1.32(\mathrm{~m}, 2 \mathrm{H}), 1.05$ (d, $J=6.8 \mathrm{~Hz}, 3 \mathrm{H}), 1.02(\mathrm{~d}, J=6.8 \mathrm{~Hz}, 3 \mathrm{H}), 0.92(\mathrm{~d}, J=6.9 \mathrm{~Hz}, 3 \mathrm{H}), 0.89(\mathrm{~d}, J=6.8 \mathrm{~Hz}, 3 \mathrm{H}), 0.86$ $(\mathrm{d}, J=6.1 \mathrm{~Hz}, 3 \mathrm{H}) ;{ }^{13} \mathrm{C}$ NMR $\left(125 \mathrm{MHz}, \mathrm{CD}_{3} \mathrm{CN}\right) \delta 196.1,157.4,137.8,137.6,137.2,135.0$, 133.3, 133.0, 132.9, 132.5, 132.3, 129.8, 129.7, 129.6, 129.5, 128.33, 128.30, 127.7, 127.4, 117.6, 79.3, 78.4, 75.0, 37.3, 36.9, 35.2, 34.5, 33.6, 33.1, 21.8, 17.9, 17.2, 17.1, 14.5, 7.9; HRMS $\left(\mathrm{ES}^{+}\right) \mathrm{m} / \mathrm{z} 610.3525\left[(\mathrm{M}+\mathrm{Na})^{+} ;\right.$calc'd for $\left.\mathrm{C}_{37} \mathrm{H}_{49} \mathrm{NNaO}_{5}: 610.3508\right]$.

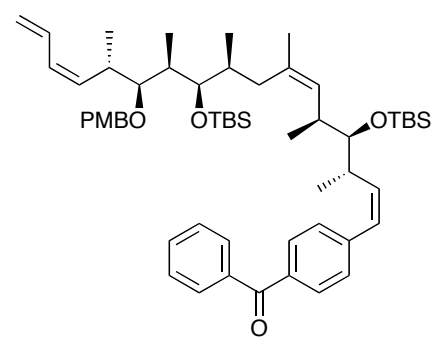

$(+)-19$

Tetraene (+)-19: Procedure For Solid Template Wittig Reaction: To a suspension of ZnO (35.0 $\mathrm{mg}, 0.432 \mathrm{mmol}), \mathrm{Cs}_{2} \mathrm{CO}_{3}(40.0 \mathrm{mg}, 0.123 \mathrm{mmol}), \mathrm{K}_{2} \mathrm{CO}_{3}(5.0 \mathrm{mg}, 0.036 \mathrm{mmol})$, and aldehyde 17 (50.0 mg, $0.238 \mathrm{mmol}$ ) in $0.5 \mathrm{~mL}$ of 1,4 dioxane was added a solution of phosphonium salt (+)15 (8:1 mixture of $Z: E$ diene isomers, $47.0 \mathrm{mg}, 0.0432 \mathrm{mmol}$ ) in 1,4 dioxane $(0.7 \mathrm{~mL})$. The 
reaction vessel was heated to $48^{\circ} \mathrm{C}$ in a resealable tube and maintained for $3 \mathrm{~h}$. The suspension was allowed to cool to rt, settle for $1 \mathrm{~h}$, and then the suspension was filtered through a plug of celite $(0.5 \times 3 \mathrm{~cm})$. The resulting plug was rinsed with EtOAc $(4 \mathrm{ml})$. This resulting solution concentrated to one quarter volume and applied directly to preparatory TLC $\mathrm{SiO}_{2}$ chromatography (Merck, $0.5 \mathrm{~mm}, 1: 10 \mathrm{EtOAc/hexanes).} \mathrm{The} \mathrm{resulting} \mathrm{product} \mathrm{band} \mathrm{was} \mathrm{cut} \mathrm{and}$ the $\mathrm{SiO}_{2}$ was rinsed with $\mathrm{EtOAc}(20 \mathrm{~mL})$ to furnish $(+)-19(31.5 \mathrm{mg}, 82 \%$ yield $)$ as a foam.

Alternative Procedure: To a $-30^{\circ} \mathrm{C}$ solution of previously dried phosphonioum salt (+)-15 (12 mg, $0.011 \mathrm{mmol}, 12: 1$ ratio of $Z / E$ diene isomers $)$ in THF (0.4 mL) was added a solution of NaHMDS (1.0 M, THF, $0.015 \mathrm{~mL}, 0.015 \mathrm{mmol}$ ) to furnish the intermediate ylide that was warmed to $-10{ }^{\circ} \mathrm{C}$ for $10 \mathrm{~min}$, recooled to $-20^{\circ} \mathrm{C}$ and a solution of aldehyde $17(14.0 \mathrm{mg}, 0.056 \mathrm{mmol})$ in $\mathrm{THF}(0.40$ $\mathrm{mL}$ ) was added. The reaction was maintained for $30 \mathrm{~min}$. at $-20^{\circ} \mathrm{C}$, allowed to warm to $0{ }^{\circ} \mathrm{C}$, and was maintained for $1 \mathrm{~h}$ followed by the addition of a saturated solution of $\mathrm{NH}_{4} \mathrm{Cl}(1.0 \mathrm{~mL})$. The emulsion was extracted with EtOAc $(3 \times 5 \mathrm{~mL})$ and the combined organics were dried $\left(\mathrm{Na}_{2} \mathrm{SO}_{4}\right)$, filtered and concentrated. Subjection of the crude residue to dried $\mathrm{SiO}_{2}$ chromatography hexanes/EtOAc (9:1) furnished (+)-19 (3.8 mg, 38\% yield). [ $[\alpha]_{\mathrm{D}}^{23}+73\left(c 1.0, \mathrm{CH}_{2} \mathrm{Cl}_{2}\right)$; IR (film, $\mathrm{NaCl}) 2960,2929,2859,1665,1610,1513,1463,1250,1084 \mathrm{~cm}^{-1} ;{ }^{1} \mathrm{H} \mathrm{NMR}\left(500 \mathrm{MHz}, \mathrm{CDCl}_{3}\right) \delta$ $7.75(\operatorname{app~d}, J=8.1 \mathrm{~Hz}, 2 \mathrm{H}), 7.73(\operatorname{app~d}, J=8.2 \mathrm{~Hz}, 2 \mathrm{H}), 7.56($ app t, $J=7.3 \mathrm{~Hz}, 1 \mathrm{H}), 7.45$ (app t, $J=7.8 \mathrm{~Hz}, 2 \mathrm{H}), 7.36(\mathrm{~d}, J=8.2 \mathrm{~Hz}, 2 \mathrm{H}), 7.26(\mathrm{~d}, J=8.2 \mathrm{~Hz}, 2 \mathrm{H}), 6.82(\mathrm{~d}, J=8.3 \mathrm{~Hz}, 2 \mathrm{H}), 6.55$ (ddd, $J=16.9,10.4,10.3 \mathrm{~Hz}, 1 \mathrm{H}), 6.38(\mathrm{~d}, J=12.0 \mathrm{~Hz}, 1 \mathrm{H}$ ), 6.03 (app t, $J=11.0 \mathrm{~Hz}, 1 \mathrm{H}$ ), 5.85 (dd, $J=11.7,10.5 \mathrm{~Hz}, 1 \mathrm{H}), 5.59(\operatorname{app~t}, J=10.4 \mathrm{~Hz}, 1 \mathrm{H}), 5.20(\mathrm{~d}, J=15.5 \mathrm{~Hz}, 1 \mathrm{H}), 5.06(\mathrm{~d}, J=$ $12.5 \mathrm{~Hz}, 1 \mathrm{H}), 4.96(\mathrm{~d}, J=9.5 \mathrm{~Hz}, 1 \mathrm{H}), 4.52\left(\mathrm{ABq}, J_{\mathrm{AB}}=10.5 \mathrm{~Hz}, v \mathrm{AB}=32.6 \mathrm{~Hz}, 2 \mathrm{H}\right), 3.78(\mathrm{~s}$, 3H), 3.40 (app t, $J=2.6 \mathrm{~Hz}, 1 \mathrm{H}), 3.32$ (dd, $J=7.0,3.6 \mathrm{~Hz}, 1 \mathrm{H}), 3.23$ (dd, $J=7.5,3.5 \mathrm{~Hz}, 1 \mathrm{H}$ ), 3.10-3.01 (m, 1H), 2.98-2.91 (m, 1H), $2.34(\operatorname{app~dt,~} J=10.0,6.8 \mathrm{~Hz}, 1 \mathrm{H}), 2.11-2.02(\mathrm{~m}, 1 \mathrm{H}), 1.98$ (app t, $J=12.3 \mathrm{~Hz}, 1 \mathrm{H}), 1.82-1.58(\mathrm{~m}, 2 \mathrm{H}), 1.53(\mathrm{~s}, 3 \mathrm{H}), 1.10(\mathrm{~d}, J=7.0 \mathrm{~Hz}, 3 \mathrm{H}), 1.05$ (d, $J=6.9$ $\mathrm{Hz}, 3 \mathrm{H}), 0.97(\mathrm{~d}, J=6.9 \mathrm{~Hz}, 3 \mathrm{H}), 0.93(\mathrm{~s}, 9 \mathrm{H}), 0.91(\mathrm{~s}, 9 \mathrm{H}), 0.86(\mathrm{~d}, J=6.7 \mathrm{~Hz}, 3 \mathrm{H}), 0.67(\mathrm{~d}, J=$ $6.7 \mathrm{~Hz}, 3 \mathrm{H}), 0.09(\mathrm{~s}, 3 \mathrm{H}), 0.08(\mathrm{~s}, 3 \mathrm{H}), 0.05(\mathrm{~s}, 3 \mathrm{H}), 0.04(\mathrm{~s}, 3 \mathrm{H}) ;{ }^{13} \mathrm{C} \mathrm{NMR}\left(125 \mathrm{MHz}, \mathrm{CDCl}_{3}\right) \delta$ 
$196.1,158.9,142.2,137.4,135.2,134.4,132.5,132.1,132.0,131.2,130.8,130.5,130.0,129.8$ $129.0,128.7,128.3,128.1,127.5,117.5,113.6,84.6,80.9,75.0,68.1,55.2,40.0,38.7,37.0$, $36.8,35.3,35.1,30.3,28.8,26.3,26.2,23.7,18.6,18.5,17.5,15.0,10.4,-3.2,-3.3,-3.4,-3.6$; HRMS (ES $\left.{ }^{+}\right) m / z 915.5754\left[(\mathrm{M}+\mathrm{Na})^{+}\right.$; calc'd for $\left.\mathrm{C}_{56} \mathrm{H}_{84} \mathrm{NaO}_{5} \mathrm{Si}_{2}: 915.5755\right]$.

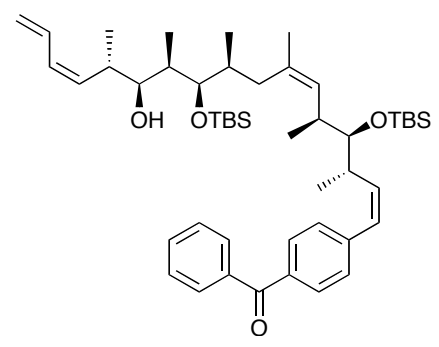

$(+)-S 4$

Alcohol (+)-S4: An emulsion of PMB ether (+)-19 (51.0 mg, $0.0571 \mathrm{mmol})$ in $\mathrm{CH}_{2} \mathrm{Cl}_{2}(4.8 \mathrm{~mL})$ and of $\mathrm{PH}-7$ buffer $(0.8 \mathrm{~mL})$ was treated with DDQ $(42.0 \mathrm{mg}, 0.185 \mathrm{mmol})$ at $0{ }^{\circ} \mathrm{C}$. After $2.5 \mathrm{~h}$ the reaction mixture was quenched with $\mathrm{NaHCO}_{3}$ saturated solution $(1.0 \mathrm{~mL})$ and extracted with EtOAc $(3 \times 25 \mathrm{~mL})$. The combined organics were washed with additional $\mathrm{NaHCO}_{3}$ saturated solution $(10 \mathrm{~mL})$, dried $\left(\mathrm{Na}_{2} \mathrm{SO}_{4}\right)$, filtered and concentrated. The residue was subjected to $\mathrm{SiO}_{2}$ chromatography $\left(9: 1\right.$ hexanes/EtOAc) to furnish alcohol (+)-S4 $\left(39.0 \mathrm{mg}, 88 \%\right.$ yield). $[\alpha]_{\mathrm{D}}^{23}+82$ (c 0.1, $\mathrm{CH}_{2} \mathrm{Cl}_{2}$ ); IR (film, NaCl) 3445, 2923, 2853, 1657, 1602, 1601, 1461, 1256, 1083, 835 $\mathrm{cm}^{-1} ;{ }^{1} \mathrm{H}$ NMR $\left(500 \mathrm{MHz}, \mathrm{CDCl}_{3}\right) \delta 7.77(\operatorname{app~d}, J=7.1 \mathrm{~Hz}, 2 \mathrm{H}), 7.76(\mathrm{~d}, J=6.7 \mathrm{~Hz}, 2 \mathrm{H}), 7.57$ (app t, $J=7.5 \mathrm{~Hz}, 1 \mathrm{H}), 7.48(\operatorname{app~t}, J=7.9 \mathrm{~Hz}, 2 \mathrm{H}), 7.38(\mathrm{~d}, J=8.3 \mathrm{~Hz}, 2 \mathrm{H}), 6.62$ (ddd, $J=16.8$, 10.7, $10.5 \mathrm{~Hz}, 1 \mathrm{H}), 6.40(\mathrm{~d}, J=12.0 \mathrm{~Hz}, 1 \mathrm{H}), 6.14(\operatorname{app~t}, J=11.0 \mathrm{~Hz}, 1 \mathrm{H}), 5.86(\mathrm{dd}, J=11.8$, $10.5 \mathrm{~Hz}, 1 \mathrm{H}), 5.37($ app t, $J=10.5 \mathrm{~Hz}, 1 \mathrm{H}), 5.22(\mathrm{~d}, J=14.8 \mathrm{~Hz}, 1 \mathrm{H}), 5.14(\mathrm{~d}, J=11.9 \mathrm{~Hz}, 1 \mathrm{H})$, $4.95(\mathrm{~d}, J=10.1 \mathrm{~Hz}, 1 \mathrm{H}), 3.56(\mathrm{dd}, J=5.5,3.4 \mathrm{~Hz}, 1 \mathrm{H}), 3.35(\mathrm{dd}, J=6.7,3.9 \mathrm{~Hz}, 1 \mathrm{H}), 3.32-3.29$ (m, 1H), 3.08 (ddd, $J=10.1,7.3,3.9 \mathrm{~Hz}, 1 \mathrm{H}), 2.79$ (app dt, $J=10.0,7.1 \mathrm{~Hz}, 1 \mathrm{H}), 2.44$ (ddd, $J=$ 10.2, 10.1, $6.8 \mathrm{~Hz}, 1 \mathrm{H}), 2.12(\operatorname{app~t}, J=12.4 \mathrm{~Hz}, 1 \mathrm{H}), 1.81-1.72(\mathrm{~m}, 3 \mathrm{H}), 1.52(\mathrm{~s}, 3 \mathrm{H}), 1.35-1.30$ (m, 1H), 1.06 (d, J = 7.0 Hz, 3H), 0.94 (d, J=6.7 Hz, 3H), 0.91 (d, J=6.5 Hz, 3H), $0.92(\mathrm{~s}, 9 \mathrm{H})$, $0.89(\mathrm{~d}, J=6.1 \mathrm{~Hz}, 3 \mathrm{H}) 0.87(\mathrm{~s}, 9 \mathrm{H}), 0.69$ (d, J = 6.9 Hz, 3H), 0.09 (s, 3H), 0.08 (s, 3H), 0.05 (s, 
3H), 0.04 (s, 3H); ${ }^{13} \mathrm{C}$ NMR (125 MHz, $\left.\mathrm{CDCl}_{3}\right) \delta 196.2,142.2,137.9,137.5,134.5,132.6,132.1$, $132.0,130.9,130.6,130.1,129.84,129.80,128.3,128.1,127.5,118.4,80.9,78.6,76.2,40.0$, $38.1,36.9,36.2,36.0,34.6,26.2,26.1,22.7,18.7,18.5,18.4,17.4,17.2,13.5,9.3,-3.2,-3.4,-$ 3.6, -3.7; HRMS $\left(\mathrm{ES}^{+}\right) \mathrm{m} / \mathrm{z} 795.5225\left[(\mathrm{M}+\mathrm{Na})^{+}\right.$; calc'd for $\left.\mathrm{C}_{48} \mathrm{H}_{76} \mathrm{NaO}_{4} \mathrm{Si}_{2}: 795.5180\right]$.

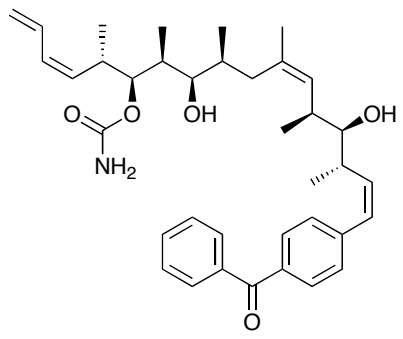

$(+)-21$

Carbamate (+)-21: A solution of alcohol (+)-S4 $(36.0 \mathrm{mg}, 0.0466 \mathrm{mmol})$ was treated with $\mathrm{Cl}_{3} \mathrm{CCON}=\mathrm{C}=\mathrm{O}(1.0 \mathrm{M}$ benzene, $0.21 \mathrm{~mL}, 0.21 \mathrm{mmol})$ and stirred for 30 minutes before direct loading onto neutral $\mathrm{Al}_{2} \mathrm{O}_{3}(1.0 \times 4.0 \mathrm{~cm}$ plug $)$. After $4 \mathrm{~h}$, the material was flushed from the $\mathrm{Al}_{2} \mathrm{O}_{3}$ (EtOAc/MeOH, 15:1, $20 \mathrm{~mL}$ ), concentrated and subsequently diluted with $0.8 \mathrm{~mL} \mathrm{MeOH}$. To the resulting solution was added aqueous hydrochloric acid $(3 \mathrm{~N}, 1.0 \mathrm{~mL})$ in $0.05 \mathrm{~mL}$ portions over 4 hours at a rate which minimized precipitation (ca. 10 to 15 min intervals). An additional $0.1 \mathrm{~mL}$ of $3 \mathrm{~N}$ aq HCl was added over $2 \mathrm{~h}$ at 15 min intervals, and the sides of the flask/stir bar were rinsed with $1.5 \mathrm{~mL}$ of $\mathrm{MeOH}$. After $14 \mathrm{~h}$ total time, an additional $0.3 \mathrm{~mL}$ of $3 \mathrm{~N}$ aq HCl was added in one portion, and the resulting solution was stirred for an additional $2 \mathrm{~h}$ at $\mathrm{rt}$. At this time the reaction was diluted with $15 \mathrm{~mL}$ of EtOAc and the reaction mixture was slowly quenched with solid $\mathrm{NaHCO}_{3}(80 \mathrm{mg})$ until cessation of bubbling. The solution was then filtered through celite and rinsed with an additional $10.0 \mathrm{~mL}$ of EtOAc. The organic layer was dried $\left(\mathrm{Na}_{2} \mathrm{SO}_{4}\right)$, filtered and concentrated. The resulting residue was subjected to $\mathrm{SiO}_{2}$ chromatography $\left(95: 5 \mathrm{CH}_{2} \mathrm{Cl}_{2} / \mathrm{MeOH}\right)$ to provide (+)-21 (19.0 mg, 69\% yield). $[\alpha]_{\mathrm{D}}^{23}+181$ (c 0.10, $\left.\mathrm{C}_{6} \mathrm{D}_{6}\right)$; IR (film, $\left.\mathrm{NaCl}\right) 3460,2963$, 2827, 1717 (broad), 1654, 1601, 1457, 1319, 1279, 1039, $978 \mathrm{~cm}^{-1} ;{ }^{1} \mathrm{H}$ NMR (500 MHz, C6 6 6) $\delta$ $7.92(\mathrm{~d}, J=7.9 \mathrm{~Hz}, 2 \mathrm{H}), 7.82(\mathrm{~d}, J=8.2 \mathrm{~Hz}, 2 \mathrm{H}), 7.46(\mathrm{~d}, J=8.2 \mathrm{~Hz}, 2 \mathrm{H}), 7.23-7.19(\mathrm{~m}, 1 \mathrm{H}), 7.18$ 
(app t, $J=7.5 \mathrm{~Hz}, 2 \mathrm{H}$ ), 6.68 (ddd, $J=16.8,11.0,10.8 \mathrm{~Hz}, 1 \mathrm{H}$ ), $6.44(\mathrm{~d}, J=12.0 \mathrm{~Hz}, 1 \mathrm{H}$ ), 6.17 (app t, $J=11.0 \mathrm{~Hz}, 1 \mathrm{H}), 5.89(\mathrm{dd}, J=11.7,10.8 \mathrm{~Hz}, 1 \mathrm{H}), 5.58($ app t, $J=10.8 \mathrm{~Hz}, 1 \mathrm{H}), 5.26$ (dd, $J=16.5,2.0 \mathrm{~Hz}, 1 \mathrm{H}), 5.13-5.08(\mathrm{~m}, 2 \mathrm{H}), 4.28-4.05(\mathrm{br} \mathrm{s}, 2 \mathrm{H}), 3.38$ (dd, $J=6.0,5.3 \mathrm{~Hz}, 1 \mathrm{H}), 3.29-$ $3.19(\mathrm{~m}, 1 \mathrm{H}), 3.14(\mathrm{dd}, J=7.0,4.3 \mathrm{~Hz}, 1 \mathrm{H}), 3.06$ (app dt, $J=13.7,6.5 \mathrm{~Hz}, 1 \mathrm{H}), 2.69$ (app dt, $J=$ 13.8, $6.9 \mathrm{~Hz}, 1 \mathrm{H}), 2.16-2.05(\mathrm{~m}, 1 \mathrm{H}), 2.02-1.90(\mathrm{~m}, 2 \mathrm{H}), 1.80(\mathrm{dd}, J=13.3,5.9 \mathrm{~Hz}, 1 \mathrm{H}), 1.67$ (s, $3 \mathrm{H}), 1.71-1.65(\mathrm{~m}, 1 \mathrm{H}), 1.42-1.38(\mathrm{~m}, 1 \mathrm{H}), 1.13(\mathrm{~d}, J=7.0 \mathrm{~Hz}, 3 \mathrm{H}), 1.11(\mathrm{~d}, J=7.0 \mathrm{~Hz}, 3 \mathrm{H}), 1.07$ $(\mathrm{d}, J=6.7 \mathrm{~Hz}, 3 \mathrm{H}), 1.02(\mathrm{~d}, J=6.8 \mathrm{~Hz}, 3 \mathrm{H}), 0.99(\mathrm{~d}, J=6.5 \mathrm{~Hz}, 3 \mathrm{H}) ;{ }^{13} \mathrm{C} \mathrm{NMR}\left(125 \mathrm{MHz}, \mathrm{C}_{6} \mathrm{D}_{6}\right)$ $\delta 195.3,156.7,141.8,138.1,136.4,135.9,134.21,134.18,132.4,131.7,130.2,129.9,129.8$, $129.0,128.6,128.5,127.0,117.5,79.7,78.0,75.3,37.6,36.9,35.9,35.6,34.9,32.7,22.9,18.4$,

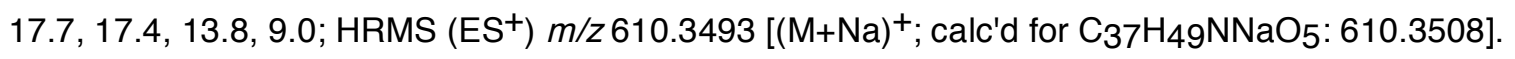

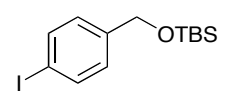

S5

4-(tert-Butyldimethylsilyloxymethyl)iodobenzene S5: To a solution of commercially available 4-iodobenzyl alcohol $(3.31 \mathrm{~g}, 14.1 \mathrm{mmol})$ in THF $(20.0 \mathrm{~mL})$ was sequentially added imidazole $(1.62 \mathrm{~g}, 23.7 \mathrm{mmol})$ and chlorodimethyltertbutylsilane $(3.24 \mathrm{~g}, 21.6 \mathrm{mmol})$. The resulting suspension was stirred at $\mathrm{rt}$ for an additional $12 \mathrm{~h}$ and was then poured into $200 \mathrm{~mL}$ of a saturated solution of $\mathrm{NaHCO}_{3}$. The resulting emulsion was diluted with EtOAc $(1000 \mathrm{~mL})$ and the bilayer was separated. The organic extract was further washed with water $(3 \times 100 \mathrm{~mL})$, dried ( $\left.\mathrm{Na}_{2} \mathrm{SO}_{4}\right)$, filtered and concentrated. The crude oil was maintained under vacuum $(1 \mathrm{~mm} \mathrm{Hg})$ for $12 \mathrm{~h}$ to furnish S5 (4.60 g, 94\% yield) of a low melting solid that was typically utilized without further purification. $\mathrm{Mp}=38-39{ }^{\circ} \mathrm{C}$; IR (film, $\mathrm{NaCl}$ ) 2954, 2928, 2884, 2856, 1590, 1483, 1462 , 1256, 1086, $839 \mathrm{~cm}^{-1} ;{ }^{1} \mathrm{H}$ NMR $\left(500 \mathrm{MHz}, \mathrm{CDCl}_{3}\right) \delta 7.67(\mathrm{~d}, J=7.9 \mathrm{~Hz}, 2 \mathrm{H}), 7.08(\mathrm{~d}, J=6.9 \mathrm{~Hz}$, 2H), $\left.4.68(\mathrm{~s}, 2 \mathrm{H}), 0.93(\mathrm{~s}, 9 \mathrm{H}), 0.10(\mathrm{~s}, 6 \mathrm{H}) ;{ }^{13} \mathrm{C} \mathrm{NMR} \mathrm{(125} \mathrm{MHz,} \mathrm{CDCl}_{3}\right) \delta$ 141.1, 137.2, 127.9, 
92.0, 64.3, 25.9, 19.1, -5.3; HRMS $\left(\mathrm{Cl}^{+}\right) \mathrm{m} / \mathrm{z} 290.9711\left[(\mathrm{M}-\mathrm{tBu})^{+}\right.$; calc'd for $\mathrm{C}_{9} \mathrm{H}_{12} \mathrm{IOSi}$ : 290.9702]. Anal. Calc'd for $\mathrm{C}_{13} \mathrm{H}_{21}$ IOSi: C, 44.83; H, 6.08. Found: $\mathrm{C}, 45.22 ; \mathrm{H}, 6.41$.

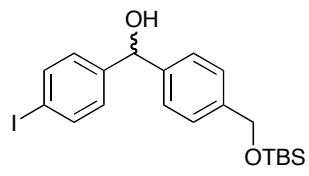

rac-24

Alcohol rac-24: A solution of silyl ether $\mathbf{S 5}(800 \mathrm{mg}, 2.3 \mathrm{mmol})$ in diethyl ether $(8.0 \mathrm{~mL})$ was cooled to $-78{ }^{\circ} \mathrm{C}$ under $\mathrm{Ar}$ and maintained for $15.0 \mathrm{~min}$. To this solution was added dropwise a solution of $t$-butyl lithium (1.5 M pentane,1.5 mL, $2.3 \mathrm{mmol})$. The resulting yellow solution was maintained for $5 \mathrm{~min}$ at $-78{ }^{\circ} \mathrm{C}$, warmed to $-40{ }^{\circ} \mathrm{C}$ for $3 \mathrm{~min}$, and recooled to $-78{ }^{\circ} \mathrm{C}$. Next, a solution of the commercially available 4-iodobenzyl aldehyde $(23,320 \mathrm{mg}, 1.4 \mathrm{mmol})$ in dry diethyl ether $(2.0 \mathrm{~mL})$ was added dropwise. After 2 minutes the reaction was warmed to $-50{ }^{\circ} \mathrm{C}$ and maintained for $3 \mathrm{~min}$. At this time the reaction was quenched with a saturated aqueous $\mathrm{NH}_{4} \mathrm{Cl}$ solution $(30 \mathrm{~mL})$ and the resulting biphasic mixture was extracted with EtOAc $(3 \times 100$ $\mathrm{mL})$. The resulting combined organics were dried $\left(\mathrm{Na}_{2} \mathrm{SO}_{4}\right)$, filtered and concentrated. The residue was subjected to $\mathrm{SiO}_{2}$ purification (9:1 EtOAc/hexanes) to furnish rac-24 (520 $\mathrm{mg}, 82 \%$ yield) as a viscous foam. IR (film, $\mathrm{NaCl}$ ) $3310,2983,2903,1587,1282,1222,836 \mathrm{~cm}^{-1} ;{ }^{1} \mathrm{H}$ NMR (500 MHz, $\left.\mathrm{CDCl}_{3}\right) \delta 7.57(\mathrm{~d}, J=8.2 \mathrm{~Hz}, 2 \mathrm{H}), 7.29(\mathrm{br} \mathrm{s}, 4 \mathrm{H}) 7.12(\mathrm{~d}, J=7.3 \mathrm{~Hz}, 2 \mathrm{H}), 5.78(\mathrm{~s}, 1 \mathrm{H})$, $4.72(\mathrm{~s}, 2 \mathrm{H}), 2.14(\mathrm{br} \mathrm{s}, 1 \mathrm{H}), 0.94(\mathrm{~s}, 9 \mathrm{H}), 0.08(\mathrm{~s}, 6 \mathrm{H}) ;{ }^{13} \mathrm{C} \mathrm{NMR}\left(125 \mathrm{MHz}, \mathrm{CDCl}_{3}\right) \delta$ 143.4, 142.0, 141.2, 137.4, 128.4, 126.4, 126.3, 92.9, 75.5, 64.6, 25.9, 18.3, -5.3; HRMS $\left(\mathrm{Cl}^{+}\right) \mathrm{m} / \mathrm{z}$ $437.0779\left[(\mathrm{M}-\mathrm{OH})^{+}\right.$; calc'd for $\left.\mathrm{C}_{20} \mathrm{H}_{26} \mathrm{IOSi}: 437.0798\right]$.

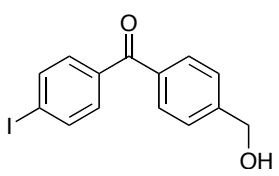


Diol S6: To a solution of silyl ether rac-24 $(600 \mathrm{mg}, 1.32 \mathrm{mmol})$ in acetonitrile $(10.0 \mathrm{~mL})$ was added 3 drops of $12.0 \mathrm{~N} \mathrm{HCl}$ (conc.). After $1 \mathrm{~h}$ the reaction was directly rotary evaporated and the resulting crude residue was directly subjected to $\mathrm{SiO}_{2}$ chromatography $(1: 1 \mathrm{EtOAc/hexanes)}$ to furnish S6 (402 mg, 91\% yield) as a crystalline solid. $\mathrm{Mp}=38-39{ }^{\circ} \mathrm{C}$; IR (film, $\mathrm{NaCl}$ ) 3320, 2923, 2872, 1585, 1480, 1391, 1031, 1004, $766 \mathrm{~cm}^{-1} ;{ }^{1} \mathrm{H}$ NMR $\left(500 \mathrm{MHz}, \mathrm{CDCl}_{3}\right) \delta 7.65$ (d, J=8.3 Hz, 2H), 7.34 (br s, 4H), $7.12(\mathrm{~d}, J=8.4 \mathrm{~Hz}, 2 \mathrm{H}), 5.78(\mathrm{~s}, 1 \mathrm{H}), 4.67(\mathrm{~s}, 2 \mathrm{H}) ;{ }^{13} \mathrm{C} \mathrm{NMR}(125 \mathrm{MHz}$,

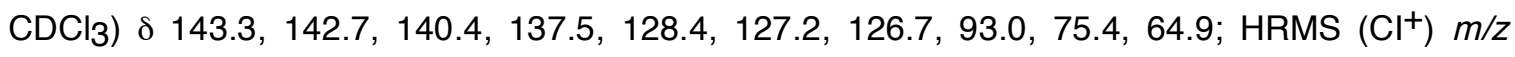
339.9947 [ $\mathrm{M}^{+}$; calc'd for $\left.\mathrm{C}_{14} \mathrm{H}_{11} \mathrm{IO}_{2}: 339.9960\right]$. Anal. Calc'd for $\mathrm{C}_{14} \mathrm{H}_{11} \mathrm{IO}_{2}: \mathrm{C}, 49.43 ; \mathrm{H}, 3.85$. Found: C, 49.61; H, 3.69.

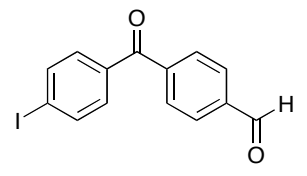

S7

Aldehyde S7. To a suspension of diol $\mathbf{S 6}(500 \mathrm{mg}, 1.47 \mathrm{mmol})$ and $\mathrm{NaHCO}_{3}(300 \mathrm{mg}, 3.57$ mmol) in $\mathrm{CH}_{2} \mathrm{Cl}_{2}(18.0 \mathrm{~mL})$ was added solid Dess-Martin periodinane reagent portionwise over $1.5 \mathrm{~h}(1.31 \mathrm{~g}, 3.10 \mathrm{mmol})$. At this time the reaction was maintained an additional $5 \mathrm{~h}$ and then diluted with EtOAc $(400 \mathrm{~mL})$. The resulting mixture was subsequently washed with $\mathrm{H}_{2} \mathrm{O}(200 \mathrm{~mL})$ and brine $(3 \times 200 \mathrm{~mL})$. The resulting organics were dried $\left(\mathrm{Na}_{2} \mathrm{SO}_{4}\right)$, filtered and concentrated. The residue was subjected to $\mathrm{SiO}_{2}$ chromatography (5:1 hexanes/ EtOAc) to furnish a white solid. The resulting solid was then dissolved in a minimum amount of EtOAc $(5 \mathrm{~mL})$ and an additional $45 \mathrm{~mL}$ of hexanes was added for purposes of recrystallizaton. After 6 hours $\mathbf{S 7}$ (378 $\mathrm{mg}, 77 \%$ yield) was collected as white crystals. $\mathrm{Mp}=139-141^{\circ} \mathrm{C}$; IR (film, $\left.\mathrm{NaCl}\right) 1694,1650$, 1580, 1391, 1300, 1204, 1111, 852, $750 \mathrm{~cm}^{-1} ;{ }^{1} \mathrm{H}$ NMR (500 MHz, CDCl $\left.{ }_{3}\right) \delta 10.12(\mathrm{~s}, 1 \mathrm{H}), 8.01$ (app d, $J=6.4 \mathrm{~Hz}, 2 \mathrm{H}), 7.89(\operatorname{app~t}, J=8.5 \mathrm{~Hz}, 4 \mathrm{H}), 7.52(\operatorname{app~d}, J=7.5 \mathrm{~Hz}, 2 \mathrm{H}) ;{ }^{13} \mathrm{C}$ NMR $(125$ $\left.\mathrm{MHz}, \mathrm{CDCl}_{3}\right) \delta 194.9,191.4,142.0,138.6,137.8,136.0,131.3,130.2,129.5,101.0 ; \mathrm{HRMS}\left(\mathrm{Cl}^{+}\right)$ 
m/z 335.9657 [ $\mathrm{M}^{+}$; calc'd for $\left.\mathrm{C}_{14} \mathrm{HgIO}_{2}: 335.9647\right]$. Anal. Calc'd for $\mathrm{C}_{14} \mathrm{HgIO}_{2}: \mathrm{C}, 50.03 ; \mathrm{H}, 2.69$. Found: $\mathrm{C}, 50.43 ; \mathrm{H}, 2.57$.

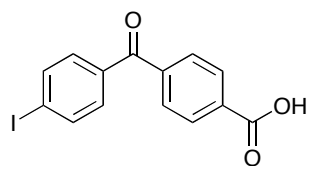

25

4-(4-lodobenzoyl) benzoic acid 25: To a solution of aldehyde $\mathbf{S 7}(1.20 \mathrm{~g}, 3.50 \mathrm{mmol})$ in $t$ - $\mathrm{BuOH}$ $(50.0 \mathrm{~mL})$ was added successively a solution of 2-methyl-2-butene (2.0 M THF, $10 \mathrm{~mL}, 20 \mathrm{mmol})$ and a solution of $\mathrm{NaH}_{2} \mathrm{PO}_{4}(290 \mathrm{mg}, 2.5 \mathrm{mmol})$ and sodium chlorite $(320 \mathrm{mg}, 2.8 \mathrm{mmol})$ in $\mathrm{H}_{2} \mathrm{O}$ $(60.0 \mathrm{~mL})$. The resulting mixture was stirred for $18.0 \mathrm{~h}$. At this time the reaction was diluted with EtOAc $(1000 \mathrm{~mL})$, washed with acid $(2.0 \mathrm{~N} \mathrm{HCl}, 600 \mathrm{~mL})$, brine $(200 \mathrm{~mL})$, and water $(2 \times 100$ $\mathrm{mL})$. The resulting organics were dried $\left(\mathrm{Na}_{2} \mathrm{SO}_{4}\right)$, filtered and concentrated to a volume of approximately $150 \mathrm{~mL}$. The solution was allowed to crystallize at $2{ }^{\circ} \mathrm{C}$ for $8 \mathrm{~h}$ to furnish white crystals. Further crystallization of the remaining mother liquor provided additional crystals for a total of $970 \mathrm{mg}\left(79 \%\right.$ yield) of $25: \mathrm{Mp}=287-289^{\circ} \mathrm{C}$; IR (film, $\left.\mathrm{NaCl}\right) 2906,1687,1645,1578,1286$, $789 \mathrm{~cm}^{-1} ;{ }^{1} \mathrm{H}$ NMR (500 MHz, D - -DMSO) $\delta 13.18(\mathrm{br} \mathrm{s}, 1 \mathrm{H}), 7.97(\mathrm{~d}, J=8.2 \mathrm{~Hz}, 2 \mathrm{H}), 7.85(\mathrm{~d}, J=$ $8.2 \mathrm{~Hz}, 2 \mathrm{H}), 7.70(\mathrm{~d}, J=8.2 \mathrm{~Hz}, 2 \mathrm{H}), 7.40(\mathrm{~d}, J=8.2 \mathrm{~Hz}, 2 \mathrm{H}) ;{ }^{13} \mathrm{C}$ NMR $\left(125 \mathrm{MHz}, \mathrm{D}_{6}-\mathrm{DMSO}\right) \delta$ 195.2, 167.0, 140.6, 138.0, 136.2, 134.6, 131.8, 130.0, 129.8, 102.0; HRMS (ES) $\mathrm{m} / \mathrm{z} 350.9508$ $\left[(\mathrm{M}-\mathrm{H})^{-} ;\right.$calc'd for $\left.\mathrm{C}_{14} \mathrm{H}_{8} \mathrm{IO}_{3}: 350.9518\right]$.

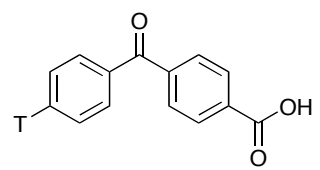

26

[3 $\mathrm{H}]$-4-benzoylbenzoic acid (26, outsourced to Perkin Elmer). A sample (100 mg, 0.286 mmol) of iodide 25 was sent to Perkin Elmer Life Sciences (New England Nuclear Division, 549 Albany St., Boston, MA, 02118). The iodide 25 was prepared as a $0.1 \mathrm{M}$ solution in DMF and 
exposed to carrier-free tritium gas using a patented priority catalyst system and pressure. Following the iodide to tritium exchange the product was doubly HPLC purified using a mobile phase of $0.1 \mathrm{TFA} / \mathrm{CH}_{3} \mathrm{CN}$ on a Zorbax SB-C18 column to furnish tritiated acid 26 which was shipped to the University of Pennsylvania as $1.0 \mathrm{mg}(117 \mathrm{mCi})$ samples in $4.5 \mathrm{~mL} \mathrm{EtOH}$ with an average specific activity of $26.2 \mathrm{Ci} / \mathrm{mmol}$. A total of 7 packaged samples $(819 \mathrm{mCi})$ of 26 were available from this process. Tritiated acid $\mathbf{2 6}$ was assayed by Perkin Elmer to be greater than 90\% chemical and radio-purity by HPLC analysis (Zorbax SB-C18 column). The compound was checked by HPLC co-injection against an the authentic commercially available cold acid $(\mathrm{T}=\mathrm{H}$, Aldrich $B, 1240-7)$. Low resolution negative ion electrospray mass spectroscopic data was also obtained for tritiated acid 26: LRMS (ES') $m / z 227.1$ [(M-H) ${ }^{-}$; calc'd for $\left.\mathrm{C}_{14} \mathrm{H}_{8} \mathrm{TO}_{3}: 227.2\right]$.

\section{Synthesis of Radiolabeled Compounds}

\section{General Procedural Notes For Radioactive Laboratory}

All reactions were run in an operating ventilation hood with an airflow set to minimum turbulence

at $120 \mathrm{feet} / \mathrm{min}$. For best containment, operator is advised to wear disposable clothing (commerically available medical scrubs) and plastic foot-covers. All floors within a 15 foot range of the hood were covered with an absorbant floor covering to prevent tracking or release of materials. All procedures including reactions, purifications by $\mathrm{SiO}_{2}$ plate or micro-column were performed in a glove bag available from Aldrich, Two-hand AtmosBags ${ }^{\top \mathrm{M}}$ cat. no Z10,608-9. Bags were equipped with lattice rods and Techware PP bench trays ${ }^{\mathrm{TM}}$ (cat. no. Z27,408-9 and Z22,5665 , respectively). Owing to the high level of tritium materials, concentration of solutions were performed with a stream of $\mathrm{Ar}$ in a glove bag rather than use of a rotary evaporator device. Likewise, water was removed during the final global deprotection procedure using repetitive concentration from EtOH under water aspirator pressure $(30 \mathrm{~mm} \mathrm{Hg})$. Sequential cold traps were utilized with the sample held in a bell jar contained within the glovebag. It was our experience that maintaining all samples in a glove bag environment minimized radioactive contamination of facilities and equipment. 


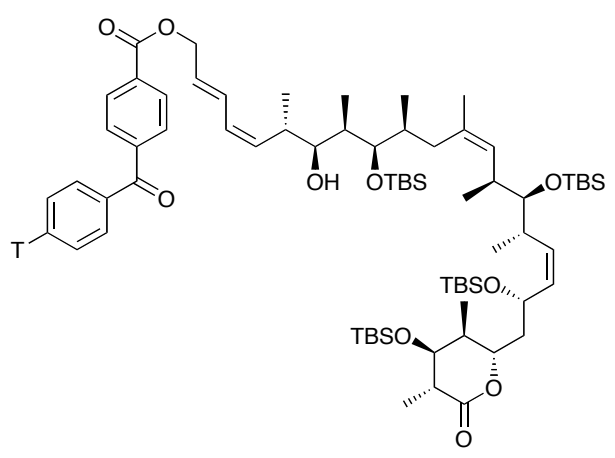

S8

Ester S8: A solution of $\left[{ }^{3} \mathrm{H}\right]$-4-benzoylbenzoic acid $26,(1.0 \mathrm{mg}, 26.5 \mathrm{Ci} / \mathrm{mmol}, 117.1 \mathrm{mCi}, 4.41$ $\mu \mathrm{mol})$ in $4.5 \mathrm{~mL}$ of EtOH was received from NEN-Perkin Elmer Life Sciences. The solution was transferred to a resealable Schlenk flask and concentrated by Ar stream. To this solid was then added $4.1 \mathrm{mg}$ of non-radioactive 4-benzoylbenzoic acid to furnish acid for reaction with a total specific activity of $5.2 \mathrm{Ci} / \mathrm{mmol}(5.1 \mathrm{mg}, 117 \mathrm{mCi}, 0.023 \mathrm{mmol})$. To this solid was added alcohol (+)-14 (11 mg, $0.011 \mathrm{mmol}), \mathrm{CH}_{2} \mathrm{Cl}_{2}(0.2 \mathrm{~mL})$, EDCI-Mel (12 mg, $\left.0.041 \mathrm{mmol}\right), \mathrm{HOBT}$ (12.0 mg, $0.089 \mathrm{mmol}$ ), and a single crystal of DMAP (approx. $1.0 \mathrm{mg}$, catalyst). The Schlenk tube was then sealed and reaction was maintained at $\mathrm{rt}$ for $18.0 \mathrm{~h}$. After complete consumption of starting material as observed by TLC analysis, the reaction contents were then directly transferred to a preparatory $\mathrm{SiO}_{2}$ plate $\left(0.25 \mathrm{~mm}\right.$, Merck) along with an additional $0.15 \mathrm{~mL} \mathrm{CH} 2 \mathrm{Cl}_{2}$ rinse. The plate was developed with EtOAc/hexanes (1:9) and the product band was cut and rinsed with EtOAc $(5 \mathrm{~mL})$ and the solvent concentrated by Ar stream to furnish $\mathbf{S 8}$ as an amorphous solid. The material was determined by serial dilution and scintillation count to be $35.2 \mathrm{mCi}$ with a specific activity of $5.2 \mathrm{Ci} / \mathrm{mmol}(8.6 \mathrm{mg}, 65 \%$ yield).

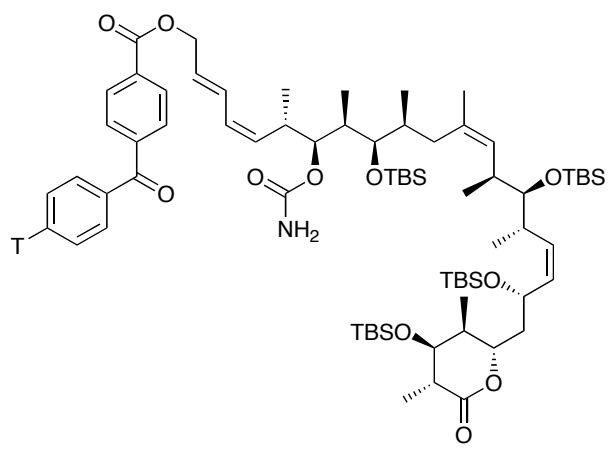


Carbamate S9 A solution of radioactive alcohol $\mathbf{S} 8$ in $\mathrm{CH}_{2} \mathrm{Cl}_{2}(0.8 \mathrm{~mL})$ was treated with $\mathrm{Cl}_{3} \mathrm{CCON}=\mathrm{C}=\mathrm{O}(20 \mu \mathrm{L}, 1.0 \mathrm{M}$ benzene solution, $0.020 \mathrm{mmol})$ at $\mathrm{rt}$ and maintained for $30 \mathrm{~min}$. The reaction mixture was loaded directly onto neutral $\mathrm{Al}_{2} \mathrm{O}_{3}(1.0 \times 4.0 \mathrm{~cm}$ plug $)$. After $4 \mathrm{~h}$, crude S9 was flushed from the $\mathrm{Al}_{2} \mathrm{O}_{3}(\mathrm{EtOAc} / \mathrm{MeOH}, 15: 1,20 \mathrm{~mL})$, concentrated by Ar stream and subsequently diluted with $0.8 \mathrm{~mL} \mathrm{MeOH}$.

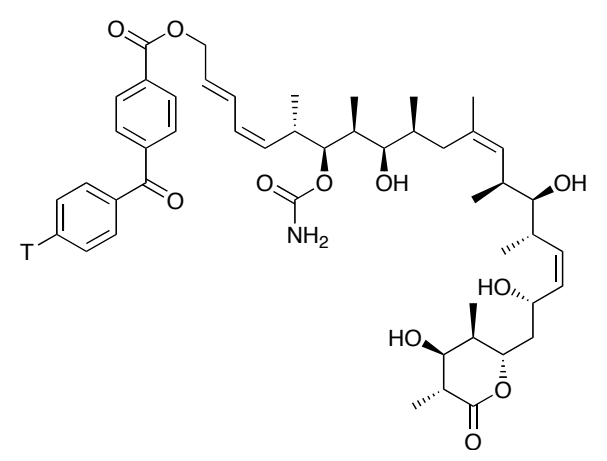

$\left[{ }^{3} \mathrm{H}\right]-10$

$\left[{ }^{3} \mathrm{H}\right]-10:$ To the resulting solution of carbamate $\mathbf{S 9}$ was added aqueous hydrochloric acid (3N, 1.0 $\mathrm{mL}$ ) in $0.05 \mathrm{~mL}$ portions over 4 hours at a rate which minimized precipitation (ca. 10 to $15 \mathrm{~min}$ intervals). An additional $0.1 \mathrm{~mL}$ of $3 \mathrm{~N}$ aq HCl was added over $2 \mathrm{~h}$ at $15 \mathrm{~min}$ intervals, and the sides of the flask/stir bar were rinsed with $1.5 \mathrm{~mL}$ of $\mathrm{MeOH}$. After $14 \mathrm{~h}$ total time, an additional $0.3 \mathrm{~mL}$ of $3 \mathrm{~N}$ aq $\mathrm{HCl}$ was added in one portion, and the resulting solution was stirred for an additional $2 \mathrm{~h}$ at $\mathrm{rt}$. At this time the reaction was diluted with $15 \mathrm{~mL}$ of EtOAc and the reaction mixture was slowly quenched with solid $\mathrm{NaHCO}_{3}(80 \mathrm{mg})$ until cessation of bubbling. The solution was then filtered through celite and rinsed with an additional $10.0 \mathrm{~mL}$ of EtOAc. The organic layer was dried ( $\left.\mathrm{Na}_{2} \mathrm{SO}_{4}\right)$, filtered, and concentrated. Residual water was removed by co-evaporation with $\mathrm{EtOH}(3 \times 3 \mathrm{~mL})$. To ensure complete removal of water, the sample was placed in a bell jar equipped with a double-cold trap and placed under aspirator vacuum (c.a. $30 \mathrm{~mm} \mathrm{Hg}$ ) for $5 \mathrm{~h}$. Immediate subjection of the crude residue to $\mathrm{SiO}_{2}$ chromatography $\left(\mathrm{CH}_{2} \mathrm{Cl}_{2} / \mathrm{MeOH} 19: 1\right.$; purified twice, micro-column $0.6 \times 4.0 \mathrm{~cm}$ plug) furnished $\left[{ }^{3} \mathrm{H}\right]-10(9.4 \mathrm{mCi}, 5.2 \mathrm{Ci} / \mathrm{mmol}, 1.5 \mathrm{mg})$. The final purity of $\left[{ }^{3} \mathrm{H}\right]-10$ was assayed by HPLC analysis, 1.0 microcurie injection samples (C-18 Zorbax 
column), and was found to be greater than 99\%. HPLC analysis was conducted with two solvent systems: $\mathrm{CH}_{3} \mathrm{CN} / \mathrm{H}_{2} \mathrm{O}$ 70:30 and $\mathrm{CH}_{3} \mathrm{CN} / \mathrm{H}_{2} \mathrm{O}$ 80:20. Additionally, mass spectroscopic and $\mathrm{UV}$ data for $\left[{ }^{3} \mathrm{H}\right]-10$ were obtained: LRMS (enriched component with T) $\left(\mathrm{ES}^{+}\right) \mathrm{m} / z$ 856.7 $\left[(\mathrm{M}+\mathrm{Na})^{+}\right.$; calc'd for $\mathrm{C}_{48} \mathrm{H}_{64} \mathrm{TNaNO}_{11}$ : 856.5]; inseparable non-radioactive component LRMS (ES $\left.{ }^{+}\right) \mathrm{m} / \mathrm{z}$ 854.7 [(M+Na) ${ }^{+}$; calc'd for $\mathrm{C}_{48} \mathrm{H}_{65} \mathrm{NaNO}_{11}$ : 854.5]; UV max: 259, $238 \mathrm{~nm}$. The final compound $(9.4 \mathrm{mCi})$ was delivered to the laboratory of Professor Susan Horwitz at Albert Einstein College of Medicine, Bronx NY as a solution in $1.92 \mathrm{~mL}$ of $\mathrm{EtOH}(0.94 \mu \mathrm{M}, 4.90 \mathrm{mCi} / \mathrm{mL}$, specific activity: 5.2 $\mathrm{Ci} / \mathrm{mmol})$.

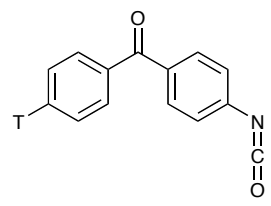

S10

Isocyanate S10: To a resealable Schlenk flask, a suspension of $\left[{ }^{3} \mathrm{H}\right]-4-$ benzoylbenzoic acid 26 $(6.3 \mathrm{mg}, 0.029 \mathrm{mmol}, 117 \mathrm{mCi}, 4.1 \mathrm{Ci} / \mathrm{mmol})^{2}$ in benzene $(0.1 \mathrm{~mL})$ was added triethylamine $(0.02$ $\mathrm{mL}, 0.14 \mathrm{mmol}$ ) at rt to furnish a clear solution. To this solution was added subsequently diphenylphosphoryl azide $(0.02 \mathrm{~mL}, d=1.28,0.093 \mathrm{mmol})$ and the flask was sealed and heated to $80{ }^{\circ} \mathrm{C}$ for $3 \mathrm{~h}$ to afford a dark-yellow solution of isocyanate $\mathbf{S 1 0}$ (ca. $0.2 \mathrm{M}$ in benzene), which was used directly in the subsequent transformations.

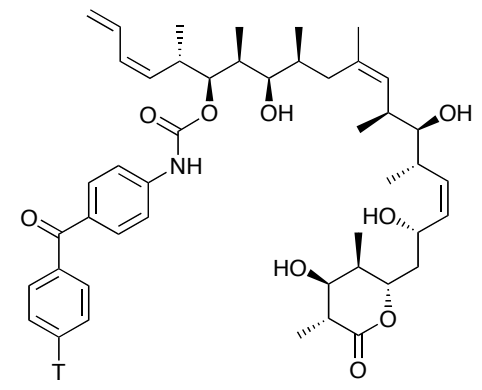

$\left[{ }^{3} \mathrm{H}\right]-13$

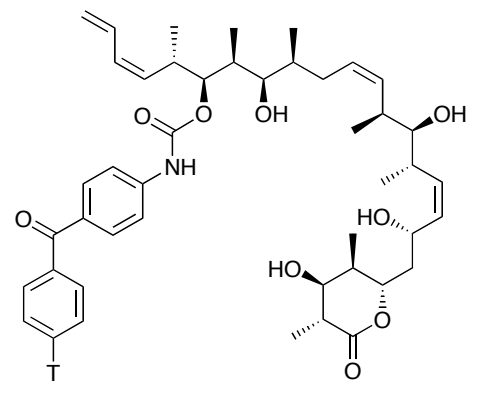

$\left[{ }^{3} \mathrm{H}\right]-14$

\footnotetext{
${ }^{2}$ The isocyanate $\mathbf{S 1 1}$ that was utilized for the synthesis of [ $\left.{ }^{3} \mathbf{H}\right]-\mathbf{1 3}$ had a specific activity of $4.1 \mathrm{Ci} / \mathrm{mmol}$ and for the synthesis of $\left[{ }^{3} \mathbf{H}\right]-\mathbf{1 4}$ had a specific activity of $4.3 \mathrm{Ci} / \mathrm{mmol}$.
} 
Radioactive Probes $\left[{ }^{3} \mathrm{H}\right]-13$ and $\left[{ }^{3} \mathrm{H}\right]-14$. Warning: the following procedure was performed in a minimum of ambient light due to the photosensitivity of the benzophenone carbamate product. To the previous solution of isocyanate $\mathbf{S 1 0}$ in a Schlenk flask was added alcohol (+)-3 $(7.0 \mathrm{mg}, 0.69$ mmol) or its $\mathrm{C}(14)$-normethyl congener $(+)-11(7.0 \mathrm{mg}, 0.68 \mathrm{mmol})$. The flask was sealed and heated to $45^{\circ} \mathrm{C}$ for $5 \mathrm{~h}$. The reaction mixture was then directly applied as a benzene solution to a Merck preparative TLC plate $(0.25 \mathrm{~mm})$ and eluted (9:1 hexanes/ EtOAc). The corresponding product band was cut and the resulting $\mathrm{SiO}_{2}$ rinsed with EtOAc $(10 \mathrm{~mL})$ to furnish the intermediate carbamate which was concentrated by Ar stream flow and diluted with $0.8 \mathrm{~mL}$ of $\mathrm{MeOH}$. To this resulting solution was added aqueous hydrochloric acid $(3 \mathrm{~N}, 1.0 \mathrm{~mL})$ in $0.04 \mathrm{~mL}$ portions over 5 hours at a rate which minimized precipitation (ca. 10 to 15 min intervals). An additional $0.4 \mathrm{~mL}$ of $3 \mathrm{~N}$ aq $\mathrm{HCl}$ was added over $2 \mathrm{~h}$ at $5 \mathrm{~min}$ intervals, and the sides of the flask/stir bar were rinsed with $0.5 \mathrm{~mL}$ of $\mathrm{MeOH}$. After $12 \mathrm{~h}$ total time, an additional $0.2 \mathrm{~mL}$ of $3 \mathrm{~N}$ aq $\mathrm{HCl}$ was added in one portion, and the resulting solution was stirred for an additional $1 \mathrm{~h}$ at $\mathrm{rt}$. At this time the reaction was diluted with $15 \mathrm{~mL}$ of EtOAc and the reaction mixture was slowly quenched with solid $\mathrm{NaHCO}_{3}(80 \mathrm{mg})$ until cessation of bubbling. The solution was then filtered through Celite and washed with an additional $10.0 \mathrm{~mL}$ of EtOAc. The organic was dried ( $\left.\mathrm{Na}_{2} \mathrm{SO}_{4}\right)$, filtered and concentrated. Residual water was removed by co-evaporation with EtOH $(3 \times 3 \mathrm{~mL})$. To ensure complete removal of water, the sample was placed in a bell jar equipped with a double-cold trap and placed under aspirator vacuum (c.a. $30 \mathrm{~mm} \mathrm{Hg}$ ) for $5 \mathrm{~h}$. Immediate subjection of the crude residue to preparatory $\mathrm{TLC} \mathrm{SiO}_{2}$ chromatography $\left(\mathrm{CH}_{2} \mathrm{Cl}_{2} / \mathrm{MeOH} 19: 1\right.$; purified in triplicate, $0.25 \mathrm{~mm}$ Merck plate) furnished $\left[{ }^{3} \mathrm{H}\right]-13(3.7 \mathrm{mCi}, 4.1 \mathrm{Ci} / \mathrm{mmol}, 0.71 \mathrm{mg})$ and in a separate but identical procedure $\left[{ }^{3} \mathrm{H}\right]-14(2.5 \mathrm{mCi}, 4.3 \mathrm{Ci} / \mathrm{mmol}, 0.44 \mathrm{mg})$.

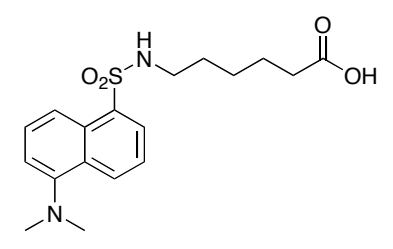


Dansylsulfonamide hexanoic acid 27: A solution of commercially available 6-aminocaproic acid (24.2 $\mathrm{mg}, 0.185 \mathrm{mmol})$, in DMF $(1.8 \mathrm{~mL})$ at room temperature, was treated with $\mathrm{N}, \mathrm{N}$ diisopropylethylamine $(161 \mu \mathrm{L}, 0.93 \mathrm{mmol})$ and trimethylsilyl chloride $(29.6 \mu \mathrm{L}, 0.23 \mathrm{mmol})$. After $10 \mathrm{~min}$, dansyl chloride $(50 \mathrm{mg}, 0.185 \mathrm{mmol}$ ) was added. After 12 hours, the reaction was quenched with an aqueous solution of $1.0 \mathrm{M} \mathrm{HCl}$ and extracted with $\mathrm{CH}_{2} \mathrm{Cl}_{2}(3 \times 10 \mathrm{~mL})$. The organic layer was dried over anhydrous $\mathrm{MgSO}_{4}$, and the product was purified by $\mathrm{SiO}_{2}$ chromatography (10:1 $\left.\mathrm{CH}_{2} \mathrm{Cl}_{2}: \mathrm{MeOH}\right)$ to afford sulfonamide 27 (30 mg, 44\% yield). IR (neat) 2925, 2359, 1700, 1456, 1318, 1143, 790, $668 \mathrm{~cm}^{-1} ;{ }^{1} \mathrm{H}$ NMR $\left(500 \mathrm{MHz}, \mathrm{CDCl}_{3}\right) \delta 8.54(\mathrm{~d}, J=8.5$ $\mathrm{Hz}, 1 \mathrm{H}), 8.29(\mathrm{~d}, J=8.6 \mathrm{~Hz}, 1 \mathrm{H}), 8.24(\mathrm{~d}, J=7.4 \mathrm{~Hz}, 1 \mathrm{H}), 7.55(\mathrm{dd}, J=8.5,7.4 \mathrm{~Hz}, 1 \mathrm{H}), 7.51$ (dd, $J=8.3,7.6 \mathrm{~Hz}, 1 \mathrm{H}), 7.19(\mathrm{~d}, J=7.6 \mathrm{~Hz}, 1 \mathrm{H}), 4.81(\operatorname{app} t, J=6.0 \mathrm{~Hz}, 1 \mathrm{H}), 2.92-2.88(\mathrm{~m}, 8 \mathrm{H}), 2.17$ (app t, $J=7.3 \mathrm{~Hz}, 2 \mathrm{H}), 1.44(\mathrm{~m}, 2 \mathrm{H}), 1.38(\mathrm{~m}, 2 \mathrm{H}), 1.19(\mathrm{~m}, 2 \mathrm{H}) ;{ }^{13} \mathrm{C} \mathrm{NMR}(125 \mathrm{MHz}, \mathrm{CDCl} 3) \delta$ $178.5,152,1,134.7,130.3,129.8,129.5,128.3,123.1,118.7,115.1,45.3,42.9,33.4,29.0,25.6$, 23.8.

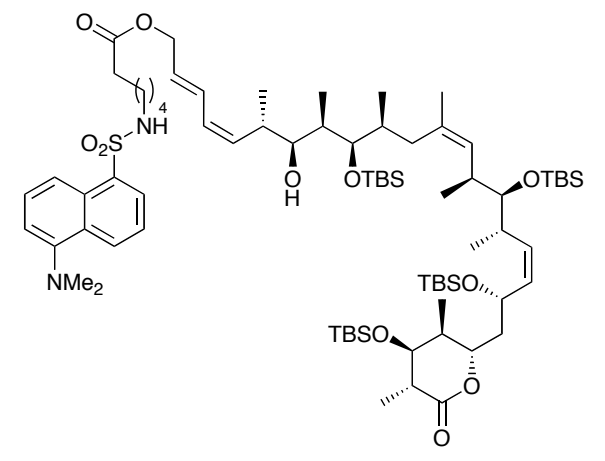

(+)-S11

Ester (+)-S11: To a resealable Schlenk tube was added diol (+)-9 (10.0 mg, $\left.9 \times 10^{-3} \mathrm{mmol}\right)$, $\mathrm{CH}_{2} \mathrm{Cl}_{2}(0.3 \mathrm{~mL})$, EDCl-Mel (10.0 mg, $\left.0.033 \mathrm{mmol}\right)$, HOBT $(8.0 \mathrm{mg}, 0.037 \mathrm{mmol})$, sulfonamide 27 (20.0 mg, $0.054 \mathrm{mmol}$ ), and a single crystal of DMAP (approx. $1.0 \mathrm{mg}$, cat.). The Schlenk tube was then sealed and reaction was maintained at rt for 12 hours until complete consumption of starting material was observed by TLC analysis. The reaction contents were then directly transferred to a preparatory $\mathrm{SiO}_{2}$ plate $\left(0.25 \mathrm{~mm}\right.$, Merck) along with an additional $0.15 \mathrm{~mL} \mathrm{CH}_{2} \mathrm{Cl}_{2}$ rinse. The plate was developed with EtOAc /hexanes (1:4) and the product band was cut and 
rinsed with EtOAc $(5 \mathrm{~mL})$ to furnish $(+)-\mathbf{S 1 1}$ as an amorphous solid $(12.0 \mathrm{mg}, 90 \%$ yield $) .[\alpha]_{\mathrm{D}}^{23}$ +28.5 (c 2.70, $\left.\mathrm{CHCl}_{3}\right)$; IR (neat) 2953, 2928, 2860, 1741, 1719, 1456, 1247, 1150, 1095, 1048, 836, $775 \mathrm{~cm}^{-1}$; ${ }^{1} \mathrm{H}$ NMR (500 MHz, $\left.\mathrm{CDCl}_{3}\right) \delta 8.54(\mathrm{~d}, J=8.5 \mathrm{~Hz}, 1 \mathrm{H}), 8.28(\mathrm{~d}, J=8.5 \mathrm{~Hz}, 1 \mathrm{H})$, $8.24(\mathrm{dd}, J=7.5,1.1 \mathrm{~Hz}, 1 \mathrm{H}), 7.56(\mathrm{dd}, J=8.5,7.5 \mathrm{~Hz}, 1 \mathrm{H}), 7.52(\operatorname{app~t}, J=8.0 \mathrm{~Hz}, 1 \mathrm{H}), 7.19$ (d, $J=7.5 \mathrm{~Hz}, 1 \mathrm{H}), 6.54(\mathrm{dd}, J=15.1,11.0 \mathrm{~Hz}, 1 \mathrm{H}), 6.12($ app t, $J=10.7 \mathrm{~Hz}, 1 \mathrm{H}), 5.75$ (app dt, $J=$ 15.1, $6.5 \mathrm{~Hz}, 1 \mathrm{H}$ ), 5.37 (app dt, $J=10.7,9.8 \mathrm{~Hz}, 1 \mathrm{H}$ ), 5.29 (dd, $J=11.1,7.9 \mathrm{~Hz}, 1 \mathrm{H}$ ), 5.19 (app t, $J=10.5 \mathrm{~Hz}, 1 \mathrm{H}), 5.08(\mathrm{~d}, J=9.9 \mathrm{~Hz}, 1 \mathrm{H}), 4.78(\operatorname{app~t}, J=9.2 \mathrm{~Hz}, 1 \mathrm{H}), 4.59(\mathrm{~m}, 3 \mathrm{H}), 4.51(\operatorname{app~t}$, $J=10.5 \mathrm{~Hz}, 1 \mathrm{H}), 3.63(\mathrm{~m}, 2 \mathrm{H}), 3.34(\mathrm{dd}, J=8.1,2.7 \mathrm{~Hz}, 1 \mathrm{H}), 3.25(\operatorname{app~t}, J=5.2 \mathrm{~Hz}, 1 \mathrm{H}), 2.94-$ $2.85(\mathrm{~m}, 8 \mathrm{H}), 2.77(\mathrm{~d}, J=7.2 \mathrm{~Hz}, 1 \mathrm{H}), 2.63(\mathrm{ddd}, J=7.5,7.5,2.7 \mathrm{~Hz}, 1 \mathrm{H}), 2.62-2.58(\mathrm{~m}, 1 \mathrm{H})$, 2.52-2.47 (m, 1H), $2.19($ app t, $J=12.3 \mathrm{~Hz}, 1 \mathrm{H}), 2.16($ app t, $J=7.4 \mathrm{~Hz}, 1 \mathrm{H}), 1.89-1.67(\mathrm{~m}, 5 \mathrm{H})$, $1.58(\mathrm{br} \mathrm{s}, 3 \mathrm{H}), 1.60-1.54(\mathrm{~m}, 1 \mathrm{H}), 1.45(\mathrm{~m}, 2 \mathrm{H}), 1.38(\mathrm{~m}, 2 \mathrm{H}), 1.22(\mathrm{~d}, J=7.5 \mathrm{~Hz}, 3 \mathrm{H}), 0.96(\mathrm{~d}, J$ $=6.3 \mathrm{~Hz}, 3 \mathrm{H}), 0.95(\mathrm{br} \mathrm{d}, J=5.5 \mathrm{~Hz}, 3 \mathrm{H}), 0.92(\mathrm{~s}, 9 \mathrm{H}), 0.90(\mathrm{~s}, 9 \mathrm{H}), 0.88(\mathrm{~s}, 9 \mathrm{H}), 0.86(\mathrm{~s}, 9 \mathrm{H})$, 0.90-0.85 (m, 9H), $0.74(\mathrm{~d}, J=6.8 \mathrm{~Hz}, 3 \mathrm{H}), 0.09(\mathrm{~s}, 6 \mathrm{H}), 0.07$ (s, 6H), $0.05(\mathrm{~s}, 6 \mathrm{H}), 0.04(\mathrm{~s}, 3 \mathrm{H})$, $0.006(\mathrm{~s}, 3 \mathrm{H}) ;{ }^{13} \mathrm{C}$ NMR $\left(125 \mathrm{MHz}, \mathrm{CDCl}_{3}\right) \delta 173.2,173.0,136.0,133.4,132.4,131.9,131.3$, 132.2, 131.1, 130.2, 129.6, 129.5, 129.4, 129.0, 128.2, 127.7, 123.3 (2), 115.2, 107.5 (2), 80.4, $78.8,76.4,74.8,64.7,64.6,45.4,44.0,42.9,42.6,37.6,37.3,36.4,36.0,35.1,35.0,34.1,33.7$, 29.6, 29.2, 26.2, 26.1, 25.8, 25.6, 24.0, 23.1, 18.3, 18.0, 17.8, 17.1, 17.0, 16.3, 15.9, 14.1, 14.0, 13.5, 9.2, -3.0, -3.3, -3.7, -4.4, -4.4, -4.5, -4.9 (2); HRMS (ES $\left.{ }^{+}\right) \mathrm{m} / \mathrm{z} 1405.867040\left[(\mathrm{M}+\mathrm{Na})^{+}\right.$; calcd for $\mathrm{C}_{75} \mathrm{H}_{134} \mathrm{~N}_{2} \mathrm{NaO}_{11} \mathrm{SSi}_{4}:$ : 1405.868320].

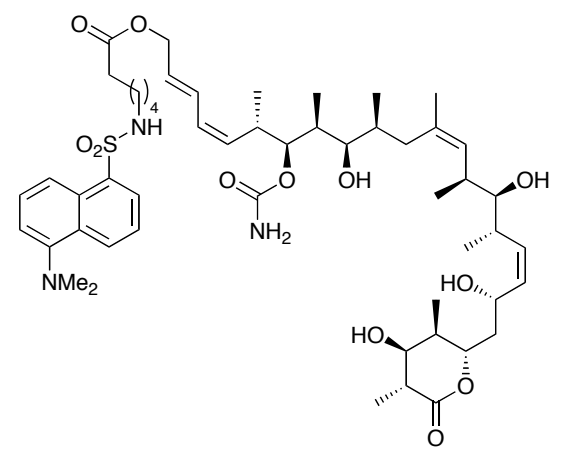

$(+)-28$ 
Ester (+)-28: A solution of alcohol (+)-S11 $(12.0 \mathrm{mg}, 0.0087 \mathrm{mmol})$ in $\mathrm{CH}_{2} \mathrm{Cl}_{2}(1.0 \mathrm{~mL})$ was treated with $\mathrm{Cl}_{3} \mathrm{CCON}=\mathrm{C}=\mathrm{O}(10.4 \mu \mathrm{L}, 1.0 \mathrm{M}$ benzene solution, $0.0104 \mathrm{mmol})$ at $\mathrm{rt}$ and maintained for $30 \mathrm{~min}$. The reaction mixture was loaded directly onto neutral $\mathrm{Al}_{2} \mathrm{O}_{3}$. After $4 \mathrm{~h}$, the material was flushed from the $\mathrm{Al}_{2} \mathrm{O}_{3}(\mathrm{EtOAc} / \mathrm{MeOH}, 15: 1,20 \mathrm{~mL})$, concentrated and diluted with $3.0 \mathrm{~mL} \mathrm{MeOH}$. To this solution was added aqueous hydrochloric acid $(3 \mathrm{~N}, 1.7 \mathrm{~mL})$ in $0.1 \mathrm{~mL}$ portions over 4 hours at a rate which minimized precipitation (ca. 10 to 15 min intervals). An additional $0.8 \mathrm{~mL}$ of $3 \mathrm{~N}$ aq HCl was added over 1 hour at 15 min intervals, and the sides of the flask/stir bar were rinsed with $1 \mathrm{~mL}$ of $\mathrm{MeOH}$. After $12 \mathrm{~h}$ total time, an additional $0.8 \mathrm{~mL}$ of $3 \mathrm{~N}$ aq HCl was added in one portion, and the resulting solution was stirred for an additional $4 \mathrm{~h}$ at $\mathrm{rt}$. At this time the reaction was diluted with $50 \mathrm{~mL}$ of EtOAc and the reaction mixture was slowly quenched with solid $\mathrm{NaHCO}_{3}(80 \mathrm{mg})$ until cessation of bubbling. The solution was then filtered through celite and washed with additional EtOAc $(50.0 \mathrm{~mL})$. The organic was dried $\left(\mathrm{Na}_{2} \mathrm{SO}_{4}\right)$, filtered and concentrated. Residual water was removed by co-evaporation with $\mathrm{EtOH}(3 \times 3 \mathrm{~mL})$. Subjection of the crude residue to $\mathrm{SiO}_{2}$ chromatography furnished (+)-28 as an amorphous solid $(1.0 \mathrm{mg}$, $65 \%$ yield). $2.0 \mathrm{mg}$ of the product of ester hydrolysis was also obtained. This compound was treated with $4 \mathrm{mg}$ of dansylaminoacid, ECDI $(2.5 \mathrm{mg}, 0.0084 \mathrm{mmol})$ of HOBT $(2.5 \mathrm{mg}, 0.018$ mmol) and a crystal of DMAP. After 10 hours the reaction mixture was transferred to a preparatory $\mathrm{SiO}_{2}$ plate $(0.25 \mathrm{~mm}$, Merck). In this fashion an additional $0.5 \mathrm{mg}$ of $(+)-28$ was obtained. $[\alpha]_{\mathrm{D}}^{23}+24.0\left(c 1.0, \mathrm{CHCl}_{3}\right)$; IR (neat) 3445, 3369, 2925, 2953, 2871, 2791, 2395, 1698, 1590, 1456, 1398, 1317, 1142, 1096, $791 \mathrm{~cm}^{-1} ;{ }^{1} \mathrm{H}$ NMR $\left(500 \mathrm{MHz}, \mathrm{CD}_{3} \mathrm{CN}\right) \delta 8.54(\mathrm{dd}, J=0.9$, $8.6 \mathrm{~Hz}, 1 \mathrm{H}), 8.28(\mathrm{~d}, J=8.7 \mathrm{~Hz}, 1 \mathrm{H}), 8.18(\mathrm{dd}, J=7.3,1.2 \mathrm{~Hz}, 1 \mathrm{H}), 7.60(\mathrm{dd}, J=8.6,7.6 \mathrm{~Hz}, 1 \mathrm{H})$, $7.58(\mathrm{dd}, J=8.5,7.4 \mathrm{~Hz}, 1 \mathrm{H}), 7.27(\mathrm{~d}, J=7.3 \mathrm{~Hz}, 1 \mathrm{H}), 6.58(\mathrm{ddd}, J=15.2,11.2,1.0 \mathrm{~Hz}, 1 \mathrm{H})$, $6.08(\operatorname{app~t}, J=11.0 \mathrm{~Hz}, 1 \mathrm{H}), 5.80(\mathrm{~m}, 1 \mathrm{H}), 5.78(\mathrm{dd}, J=15.2,5.9 \mathrm{~Hz}, 1 \mathrm{H}), 5.55(\operatorname{app~t}, J=10.7$ $\mathrm{Hz}, 1 \mathrm{H}), 5.45(\operatorname{app} t J=10.7 \mathrm{~Hz}, 1 \mathrm{H}), 5.38(\mathrm{dd}, J=10.7,8.8 \mathrm{~Hz}, 1 \mathrm{H}), 5.07(\mathrm{~m}, 2 \mathrm{H}), 4.96(\mathrm{~d}, J=$ $10.2 \mathrm{~Hz}, 1 \mathrm{H}), 4.73(\mathrm{dd}, J=8.1,4.0 \mathrm{~Hz}, 1 \mathrm{H}), 4.62(\mathrm{dd}, J=13.5,5.6 \mathrm{~Hz}, 1 \mathrm{H}), 4.55(\mathrm{dd}, J=13.5$, $5.6 \mathrm{~Hz}, 1 \mathrm{H}), 4.47$ (ddd, $J=10.6,10.6,1.7 \mathrm{~Hz}, 1 \mathrm{H}), 4.44(\mathrm{~m}, 1 \mathrm{H}), 3.63(\mathrm{~d}, J=3.0 \mathrm{~Hz}, 1 \mathrm{H}), 3.29$ (m, 1H), $3.13(\mathrm{~m}, 1 \mathrm{H}), 3.07(\mathrm{~m}, 2 \mathrm{H}), 2.87(\mathrm{~s}, 6 \mathrm{H}), 2.82(\mathrm{dd}, J=13.1,6.8 \mathrm{~Hz}, 2 \mathrm{H}), 2.76(\mathrm{~m}, 1 \mathrm{H})$, 
2.63-2.60 (m, 2H), $2.57(\mathrm{dd}, J=7.4,4.5 \mathrm{~Hz}, 1 \mathrm{H}), 2.22(\mathrm{~m}, 1 \mathrm{H}), 2.18(\mathrm{~m}, 2 \mathrm{H}), 1.85(\mathrm{~m}, 1 \mathrm{H}), 1.77-$ $1.65(\mathrm{~m}, 5 \mathrm{H}), 1.62$ (d, $J=12.8 \mathrm{~Hz}, 1 \mathrm{H}), 1.59$ (s, 3H), 1.46 (ddd, $J=14.3,10.5,2.9 \mathrm{~Hz}, 1 \mathrm{H}), 1.38$ (m, 2H), 1.30-1.26 (m, 2H), $1.19(\mathrm{~d}, J=7.3 \mathrm{~Hz}, 3 \mathrm{H}), 1.11(\mathrm{~m}, 2 \mathrm{H}), 1.01(\mathrm{~d}, J=7.2 \mathrm{~Hz}, 3 \mathrm{H}), 1.00$ (d, $J=7.1 \mathrm{~Hz}, 3 \mathrm{H}), 0.95(\mathrm{~d}, J=6.7 \mathrm{~Hz}, 3 \mathrm{H}), 0.88(\mathrm{~d}, J=6.6 \mathrm{~Hz}, 3 \mathrm{H}), 0.80(\mathrm{~d}, J=6.8 \mathrm{~Hz}, 3 \mathrm{H})$, $0.77(\mathrm{~d}, J=6.0 \mathrm{~Hz}, 3 \mathrm{H}) ;{ }^{13} \mathrm{C}$ NMR $\left(125 \mathrm{MHz}, \mathrm{CD}_{3} \mathrm{CN}\right) \delta 173.2,172.9,157.2,152.1,152.0,133.8$, 133.6, 132.8, 132.7, 130.0, 129.9, 129.6, 129.3, 129.0, 128.3, 128.0, 127.8, 123.3, 118.8, 115.1, 78.7, 78.2, 76.4, 74.9, 72.1, 63.9, 62.3, 44.6, 42.9, 42.5, 41.1, 37.4, 36.2, 35.5, 35.2 (2), 33.6, 33.3, 33.2, 29.2, 28.6, 25.4, 23.9, 22.2, 18.7, 17.1, 16.7, 14.7, 14.6, 12.0, 8.1; HRMS (ES+) m/z $992.5236\left[(\mathrm{M}+\mathrm{Na})^{+}\right.$; calcd for $\mathrm{C}_{52} \mathrm{H}_{79} \mathrm{~N}_{3} \mathrm{NaO}_{12} \mathrm{~S}:$ 992.5282].

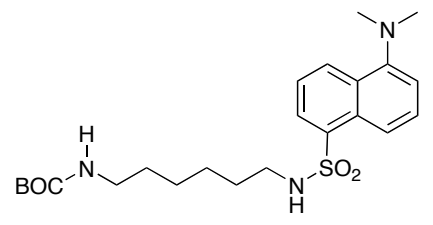

S12

N-Boc-dansylamine S12: Commercially available N-Boc-1,6-hexanediamine hydrochloride $(0.100 \mathrm{~g}, 0.395 \mathrm{mmol})$, dansyl chloride $(0.106 \mathrm{~g}, 0.395 \mathrm{mmol})$, and Hünig's base $(0.206 \mathrm{~mL}, 1.2$ mmol) were combined in a round-bottom flask and dissolved in $\mathrm{CH}_{2} \mathrm{Cl}_{2}(3 \mathrm{~mL})$. After stirring over night, the reaction was evaporated under vacuum, and the residue was purified by flash chromatography (30\% EtOAc/hexanes) to afford protected dansylamine S12 (160 mg, 90\%); IR (neat) $3335,2982,2934,2860,2791,1694,1504,1322,1250,1163,1144,1093,791 \mathrm{~cm}^{-1} ;{ }^{1} \mathrm{H}$ NMR $\left(500 \mathrm{MHz}, \mathrm{CDCl}_{3}\right) \delta 8.54(\mathrm{~d}, J=8.4 \mathrm{~Hz}, 1 \mathrm{H}), 8.29(\mathrm{~d}, J=8.6 \mathrm{~Hz}, 1 \mathrm{H}), 8.24(\mathrm{~d}, J=7.0 \mathrm{~Hz}$, 1H), $7.57(\mathrm{dd}, J=8.2,8.1 \mathrm{~Hz}, 1 \mathrm{H}), 7.53(\mathrm{dd}, J=8.0,7.8 \mathrm{~Hz}, 1 \mathrm{H}), 7.19(\mathrm{~d}, J=7.4 \mathrm{~Hz}, 1 \mathrm{H}), 5.05$ (br s, $1 \mathrm{H}), 4.52(\mathrm{br} \mathrm{s}, 1 \mathrm{H}), 3.00-2.93(\mathrm{~m}, 2 \mathrm{H}), 2.90-2.84(\mathrm{~m}, 8 \mathrm{H}), 1.41(\mathrm{~s}, 9 \mathrm{H}), 1.32$ (ddd, $J=14.1$, 7.3, 7.2 Hz, 2H), 1.23 (ddd, $J=7.1,7.0,1.8 \mathrm{~Hz}, 2 \mathrm{H}), 1.12-0.91(\mathrm{~m}, 4 \mathrm{H}) ;{ }^{13} \mathrm{C}$ NMR $(125 \mathrm{MHz}$, $\left.\mathrm{CDCl}_{3}\right) \delta 155.8,151.8,134.9,130.2,129.8,129.6,129.4,128.2,123.1,118.8,115.1,78.9,45.3$, $42.9, \quad 40.1,29.6,29.2,28.3,25.88,25.81 ;\left(\mathrm{ES}^{+}\right) \mathrm{m} / \mathrm{z} 472.223466\left[(\mathrm{M}+\mathrm{Na})^{+} ;\right.$calcd for $\left.\mathrm{C}_{23} \mathrm{H}_{35} \mathrm{~N}_{3} \mathrm{NaO}_{4} \mathrm{~S}: 472.224598\right]$. 


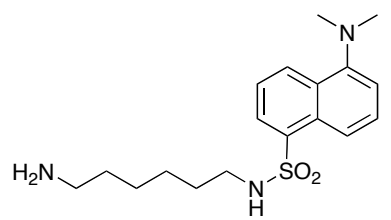

30

Dansylamine 30: To a solution of $\mathbf{S} 12(40 \mathrm{mg}, 0.16 \mathrm{mmol})$ in $\mathrm{CH}_{2} \mathrm{Cl}_{2}(0.24 \mathrm{~mL})$ was added, at rt, TFA $(0.6 \mathrm{~mL}, 16.8 \mathrm{mmol})$. The resulting solution was stirred for $1 \mathrm{~h}$ and then $\mathrm{Et}_{3} \mathrm{SiH}(0.255 \mathrm{~mL}, 16$ mmol) was added. After 4 hours, the reaction was done, and evaporation of solvents and reagents afforded 30 as a crude oil (yield $>95 \%$ base on purity of ${ }^{1} \mathrm{H}$ NMR). This amine was typically used in the next step without purification. IR (neat) $3383,3307,2982,2934,2860,2789$, $1694,1524,1455,1322,1256,1144,1070,791 \mathrm{~cm}^{-1} ;{ }^{1} \mathrm{H}$ NMR $\left(500 \mathrm{MHz}, \mathrm{CDCl}_{3}\right) \delta 8.47(\mathrm{~d}, J=$ $7.6 \mathrm{~Hz}, 1 \mathrm{H}), 8.28(\mathrm{~d}, J=8.7 \mathrm{~Hz}, 1 \mathrm{H}), 8.19(\mathrm{~d}, J=7.0 \mathrm{~Hz}, 1 \mathrm{H}), 7.50(\mathrm{dd}, J=8.4,7.6 \mathrm{~Hz}, 1 \mathrm{H}), 7.47$ (dd, $J=8.5,7.4 \mathrm{~Hz}, 1 \mathrm{H}), 7.13(\mathrm{~d}, J=7.5 \mathrm{~Hz}, 1 \mathrm{H}), 3.85-2.83(\mathrm{~m}, 8 \mathrm{H}), 2.53(\mathrm{dd}, J=6.9,6.8 \mathrm{~Hz}$, 2H), 1.32 (ddd, $J=14.3,7.1,7.1 \mathrm{~Hz}, 2 \mathrm{H}), 1.23$ (ddd, $J=14.2,6.9,6.0 \mathrm{~Hz}, 2 \mathrm{H}), 1.12-1.04(\mathrm{~m}$, $4 \mathrm{H}) ;{ }^{13} \mathrm{C}$ NMR $\left(125 \mathrm{MHz}, \mathrm{CDCl}_{3}\right) \delta 151.9,134.9,130.1,129.8,129.6,129.4,128.1,123.1,118.8$, 115.0, 45.3, 43.0, 41.6, 32.9, 29.3, 26.06, 26.01; HRMS (ES $\left.{ }^{+}\right) \mathrm{m} / \mathrm{z} 350.190087$ [(M+H) ${ }^{+}$; calcd for $\left.\mathrm{C}_{18} \mathrm{H}_{28} \mathrm{~N}_{3} \mathrm{O}_{2} \mathrm{~S}: 472.224598\right]$.

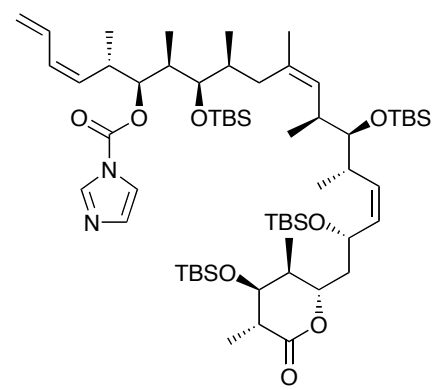

$(+)-29$

Imidazole carbamate (+)-29: 1,1'-carbonyldiimidazole $(3.6 \mathrm{mg}, 0.022 \mathrm{mmol})$ was added to a solution of alcohol $(+)-3(15 \mathrm{mg}, 0.015 \mathrm{mmol})$ in $\mathrm{CH}_{2} \mathrm{Cl}_{2}(0.15 \mathrm{~mL})$. After $12 \mathrm{~h}$ the solution was concentrated under reduced pressure and purified by flash chromatography ( $9: 1$ hexanes:EtOAc) to afford carbamate (+)-29 (9 mg, 61\% yield). $[\alpha]_{\mathrm{D}}^{23}+35^{\circ}\left(\mathrm{c} 0.2, \mathrm{CHCl}_{3}\right)$; IR (neat) 2957, 2928, 2888, 2856, 1761, 1472, 1252, 1237, 1177, 1094, 1046, $835 \mathrm{~cm}^{-1} ;{ }^{1} \mathrm{H}$ NMR $\left(500 \mathrm{MHz}, \mathrm{CDCl}_{3}\right) \delta$ 
$8.09(\mathrm{~s}, 1 \mathrm{H}), 7.38(\mathrm{~s}, 1 \mathrm{H}), 7.05(\mathrm{~s}, 1 \mathrm{H}), 6.54(\mathrm{ddd}, J=16.8,10.7,10.6 \mathrm{~Hz}, 1 \mathrm{H}), 6.03(\mathrm{dd}, J=11.0$, $10.9 \mathrm{~Hz}, 1 \mathrm{H}$ ), $5.34(\mathrm{dd}, J=10.5,10.5 \mathrm{~Hz}, 1 \mathrm{H}), 5.28(\mathrm{dd}, J=11.1,7.8 \mathrm{~Hz}, 1 \mathrm{H}), 5.21-5.14(\mathrm{~m}, 3 \mathrm{H})$, 5.08-5.05 (m, 2H), $4.78(\mathrm{dd}, J=9.2,9.2 \mathrm{~Hz}, 1 \mathrm{H}), 4.51$ (dd, $J=10.1,10.1 \mathrm{~Hz}, 1 \mathrm{H}), 3.63(\mathrm{dd}, J=$ 2.4, $2.3 \mathrm{~Hz}, 1 \mathrm{H}), 3.46(\mathrm{dd}, J=4.4,4.4 \mathrm{~Hz}, 1 \mathrm{H}), 3.23(\mathrm{dd}, J=5.4,5.3 \mathrm{~Hz}, 1 \mathrm{H}), 3.19-3.12(\mathrm{~m}, 1 \mathrm{H})$, 2.65-2.57 (m, 2H), 2.45 (ddd, $J=6.4,6.4,6.4 \mathrm{~Hz}, 1 \mathrm{H}), 2.13-2.04(\mathrm{~m}, 2 \mathrm{H}), 1.88-1.82(\mathrm{~m}, 1 \mathrm{H})$, 1.82-1.75 (m, 1H),1.74-1.61 (m, 2H), $1.56(\mathrm{~m}, 4 \mathrm{H}), 1.22(\mathrm{~d}, J=7.6 \mathrm{~Hz}, 3 \mathrm{H}), 1.06(\mathrm{~d}, J=6.8 \mathrm{~Hz}$, 3H), 1.01 (d, J =6.9 Hz, 3H), 0.95 (app d, J =6.7 Hz, 6H), 0.92 (s, 9H), 0.90 (s, 9H), 0.88 (s, 9H), 0.87-0.86 (m, 3H), $0.87(\mathrm{~s}, 9 \mathrm{H}), 0.74(\mathrm{~d}, J=6.7 \mathrm{~Hz}, 3 \mathrm{H}), 0.076(\mathrm{~s}, 3 \mathrm{H}), 0.070(\mathrm{~s}, 6 \mathrm{H}), 0.06(\mathrm{~s}$, 9H), 0.04 (s, 3H), 0.0006 (s, 3H); ${ }^{13} \mathrm{C}$ NMR $\left(125 \mathrm{MHz}, \mathrm{CDCl}_{3}\right) \delta$ 173.2, 148.7, 137.0, 133.4, 132.3, $132.1,131.4,131.0,130.8,130.7,130.4,119.3,117.0,83.5,80.3,76.9,76.8,74.8,64.6,44.1$, $42.6,37.8,37.4,35.8,35.3,35.2,34.4,34.1,26.2,26.1,25.9,25.7,22.9,18.40,18.37,17.9$, $17.8,17.5,17.1,16.4,16.2,14.1,14.0,10.3,-3.0,-3.3,-3.7,-4.42,-4.45,-4.5,-4.9$ (2C); HRMS $\left(\mathrm{ES}^{+}\right) \mathrm{m} / \mathrm{z} 1123.73447\left[(\mathrm{M}+\mathrm{Na})^{+} ;\right.$calcd for $\left.\mathrm{C}_{60} \mathrm{H}_{112} \mathrm{~N}_{2} \mathrm{NaO}_{8} \mathrm{Si}_{4}: 1123.739353\right]$.

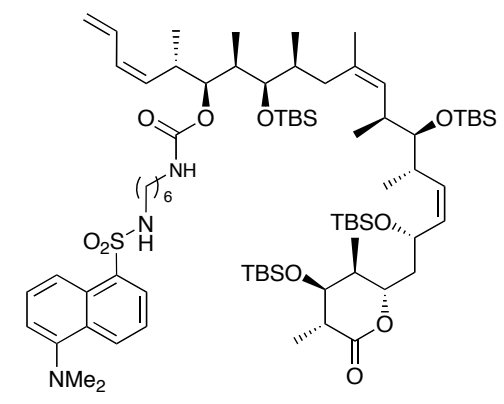

$(+)-\mathbf{S 1 3}$

Carbamate (+)-S13: To a solution of carbamate (+)-29 (10 mg, $\left.9.1 \times 10^{-3} \mathrm{mmol}\right)$ in $\mathrm{CH}_{2} \mathrm{Cl}_{2}(0.5$ $\mathrm{mL}$ ) in a sealed tube, were added DMAP and $\mathrm{Et}_{3} \mathrm{~N}$, followed by a solution of amine $\mathbf{3 0}$ (excess). The reaction was then heated at $40{ }^{\circ} \mathrm{C}$ for 4 hours and, when no starting material remained as judged by TLC analysis, the mixture was concentrated and purified by flash chromatography (10\% EtOAc/hexanes) to afford carbamate (+)-S13 $(8 \mathrm{mg}, 63 \%) \cdot[\alpha]_{\mathrm{D}}^{23}+48.0\left(c 0.1, \mathrm{CHCl}_{3}\right) ; \mathrm{IR}$ (neat) $3455,2959,2928,2855,1716,1463,1258,1095,1039,836 \mathrm{~cm}^{-1} ;{ }^{1} \mathrm{H}$ NMR $(500 \mathrm{MHz}$, 
$\left.\mathrm{CDCl}_{3}\right) \delta 8.54(\mathrm{~d}, J=8.5 \mathrm{~Hz}, 1 \mathrm{H}), 8.28(\mathrm{~d}, J=8.6 \mathrm{~Hz}, 1 \mathrm{H}), 8.24(\mathrm{dd}, J=7.3,1.0 \mathrm{~Hz}, 1 \mathrm{H}), 8.21(\mathrm{~s}$, 1H), $7.56(\mathrm{dd}, J=8.4,7.9 \mathrm{~Hz}, 1 \mathrm{H}), 7.52(\mathrm{dd}, J=8.3,7.5 \mathrm{~Hz}, 1 \mathrm{H}), 7.18(\mathrm{~d}, J=7.5 \mathrm{~Hz}, 1 \mathrm{H}), 6.58$ (ddd, $J=17.0,10.8,10.8 \mathrm{~Hz}, 1 \mathrm{H}$ ), $5.97(\mathrm{dd}, J=11.1,11.1 \mathrm{~Hz}, 1 \mathrm{H}$ ), $5.36(\mathrm{dd}, J=10.3,10.3 \mathrm{~Hz}$, 1H), 5.28 (dd, $J=11.1,7.8 \mathrm{~Hz}, 1 \mathrm{H}), 5.19(\mathrm{~d}, J=9.5 \mathrm{~Hz}, 1 \mathrm{H}), 5.17$ (d, $J=9.7 \mathrm{~Hz}, 1 \mathrm{H}), 5.11(\mathrm{~d}, J=$ $10.3 \mathrm{~Hz}, 1 \mathrm{H}), 5.04(\mathrm{~d}, J=9.7 \mathrm{~Hz}, 1 \mathrm{H}), 4.78(\mathrm{dd}, J=9.0,9.0 \mathrm{~Hz}, 1 \mathrm{H}), 4.71(\mathrm{br} \mathrm{s}, 1 \mathrm{H}), 4.53-4.15$ (m, 2H), $3.64(\mathrm{dd}, J=2.3,2.3 \mathrm{~Hz}, 1 \mathrm{H}), 3.43(\mathrm{dd}, J=4.1,3.9 \mathrm{~Hz}, 1 \mathrm{H}), 3.23(\mathrm{dd}, J=5.4,5.3 \mathrm{~Hz}$, 1H), 3.09 (s, 2H), $2.99(\mathrm{~m}, 2 \mathrm{H}), 2.89-2.82(\mathrm{~m}, 8 \mathrm{H}), 2.63$ (ddd, J = 7.7, 7.5, $2.8 \mathrm{~Hz}, 1 \mathrm{H}), 2.62-2.58$ (m, 1H), 2.45-2.40 (m, 1H), 2.05-2.00 (m, 1H), 1.90-1.55 (m, 5H), 1.40-1.20 (m, 8H), $1.25(\mathrm{~s}, 3 \mathrm{H})$, $1.22(\mathrm{~d}, J=7.6 \mathrm{~Hz}, 3 \mathrm{H}), 1.15-1.10(\mathrm{~m}, 6 \mathrm{H}), 0.97(\mathrm{~d}, J=6.7 \mathrm{~Hz}, 3 \mathrm{H}), 0.92(\mathrm{~m}, 9 \mathrm{H}), 0.92-0.91(\mathrm{~m}$, 3H), $0.90(\mathrm{~s}, 9 \mathrm{H}), 0.88(\mathrm{~s}, 9 \mathrm{H}), 0.86(\mathrm{~d}, J=6.5 \mathrm{~Hz}, 3 \mathrm{H}), 0.86(\mathrm{~s}, 9 \mathrm{H}), 0.71(\mathrm{~d}, J=6.7 \mathrm{~Hz}, 3 \mathrm{H})$, 0.08 (s, 3H), 0.07 (s, 9H), 0.05 (s, 3H), 0.05 (s, 3H), $0.04(\mathrm{~s}, 3 \mathrm{H}),-0.001(\mathrm{~s}, 3 \mathrm{H}) ;{ }^{13} \mathrm{C}$ NMR $(125$ $\left.\mathrm{MHz}, \mathrm{CDCl}_{3}\right) \delta 173.2,156.6,152.0,134.7,133.7,133.3,132.4,132.0,131.7,131.2,130.3,129.8$, 129.6, 129.5 (2), 128.3, 123.1, 118.6, 117.7, 115.1, 80.4, 78.3, 76.8, 76.2, 74.8, 64.5, 45.3 (2), $44.1,43.0,42.6,40.5,37.6,37.4,35.6,35.4,35.0,34.4,34.0,29.7,29.4,26.2,26.1,25.8,25.8$, $25.6,22.9,18.4,18.3,18.0,17.8,17.5,16.9,16.3,16.1,14.0,13.9,10.0,-3.0,-3.5,-3.6,-4.42,-$ 4.40, -4.5, -4.9 (2); HRMS (ES $\left.{ }^{+}\right) \mathrm{m} / z 1404.891902\left[(\mathrm{M}+\mathrm{Na})^{+}\right.$; calcd for $\mathrm{C}_{75} \mathrm{H}_{135} \mathrm{~N}_{3} \mathrm{NaO}_{10} \mathrm{SSi}_{4}$ : 1404.884304].

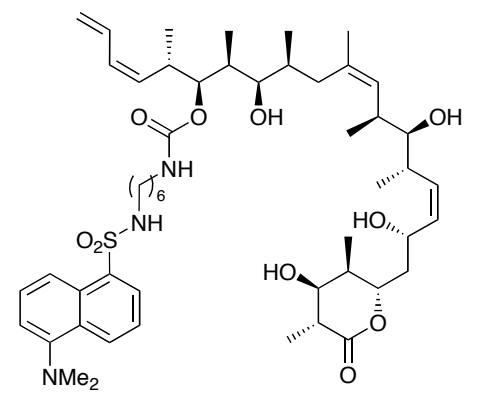

$(+)-31$

Tetraol (+)-31: Carbamate (+)-S13 (8 mg, $\left.5.7 \times 10^{-3} \mathrm{mmol}\right)$ was dissolved in $\mathrm{MeOH}(2 \mathrm{~mL})$ and stirred for $15 \mathrm{~min}$ at room temperature. Aqueous hydrochloric acid $(3 \mathrm{~N}, 1.2 \mathrm{~mL})$ was added in $0.05 \mathrm{~mL}$ portions over 4 hours at a rate which minimized precipitation (ca. 10 to $15 \mathrm{~min}$ intervals). 
An additional $0.6 \mathrm{~mL}$ of $3 \mathrm{~N}$ aq HCl was added over $1 \mathrm{~h}$ at $15 \mathrm{~min}$ intervals, and the sides of the flask/stir bar were rinsed with $1 \mathrm{~mL}$ of $\mathrm{MeOH}$. After $8 \mathrm{~h}$, an additional $0.6 \mathrm{~mL}$ of $3 \mathrm{~N}$ aq $\mathrm{HCl}$ was added in one portion, and the resulting solution was stirred for $2 \mathrm{~h}$ at $\mathrm{rt}$, diluted with $20 \mathrm{~mL}$ of EtOAc, and the resulting mixture was neutralized by addition of $\mathrm{NaHCO}_{3}$, dried with $\mathrm{MgSO}_{4}$, then filtered and concentrated. Flash chromatography using washed $\mathrm{SiO}_{2}\left(10 \% \mathrm{MeOH} / \mathrm{CH}_{2} \mathrm{Cl}_{2}\right.$ then $5 \%$ $\left.\mathrm{MeOH} / \mathrm{CH}_{2} \mathrm{Cl}_{2}\right)$ via gradient elution $\left(5 \% \mathrm{MeOH} / \mathrm{CH}_{2} \mathrm{Cl}_{2}\right.$ to $\left.10 \% \mathrm{MeOH} / \mathrm{CH}_{2} \mathrm{Cl}_{2}\right)$ gave (+)-31 (2.8 $\mathrm{mg}, 52 \%$ yield). $[\alpha]_{\mathrm{D}}^{23}+10\left(\mathrm{c} 0.1, \mathrm{CHCl}_{3}\right) ; \mathrm{IR}$ (neat) 3391, 2965, 2934, 2872, 1699, 1456, 1316, 1233, 1142, $1099 \mathrm{~cm}^{-1} ;{ }^{1} \mathrm{H}$ NMR $\left(500 \mathrm{MHz}, \mathrm{CD}_{3} \mathrm{CN}\right) \delta 8.54(\mathrm{dd}, J=8.6,0.9 \mathrm{~Hz}, 1 \mathrm{H}), 8.28(\mathrm{~d}, J=$ $8.6 \mathrm{~Hz}, 1 \mathrm{H}), 8.17(\mathrm{dd}, J=7.3,1.2 \mathrm{~Hz}, 1 \mathrm{H}), 7.60(\mathrm{dd}, J=8.6,7.6 \mathrm{~Hz}, 1 \mathrm{H}), 7.58(\mathrm{dd}, J=8.6,7.3$ $\mathrm{Hz}, 1 \mathrm{H}), 7.27(\mathrm{~d}, J=7.4 \mathrm{~Hz}, 1 \mathrm{H}), 6.66(\mathrm{ddd}, J=16.8,10.4,10.4 \mathrm{~Hz}, 1 \mathrm{H}), 6.04(\mathrm{dd}, J=11.1,11.1$ Hz, 1H), $5.79(\mathrm{dd}, J=10.4,10.1 \mathrm{~Hz}, 1 \mathrm{H}), 5.45(\mathrm{~s}, 1 \mathrm{H}), 5.42-5.36(\mathrm{~m}, 3 \mathrm{H}), 5.25(\mathrm{dd}, J=16.9,2.0$ $\mathrm{Hz}, 1 \mathrm{H}), 5.14(\mathrm{~d}, J=10.2 \mathrm{~Hz}, 1 \mathrm{H}), 4.99(\mathrm{~d}, J=10.0 \mathrm{~Hz}, 1 \mathrm{H}), 4.71$ (dd, $J=7.2,4.6 \mathrm{~Hz}, 1 \mathrm{H}), 4.46$ (ddd, $J=10.5,10.5,1.9 \mathrm{~Hz}, 1 \mathrm{H}), 4.49-4.44(\mathrm{~m}, 1 \mathrm{H}), 3.63-3.60(\mathrm{~m}, 1 \mathrm{H}), 3.28(\mathrm{~s}, 1 \mathrm{H}), 3.27(\mathrm{~s}, 1 \mathrm{H})$, 3.14-3.02 (m, 3H), 2.96-2.90 (m, 2H), $2.87(\mathrm{~s}, 6 \mathrm{H}), 2.82$ (ddd, J = 6.8, 6.8, $6.8 \mathrm{~Hz}, 2 \mathrm{H}), 2.65-2.55$ (m, 3H), 2.33-2.27 (m, 1H), 1.89-1.60 (m, 6H), $1.58(\mathrm{~d}, J=0.9 \mathrm{~Hz}, 3 \mathrm{H}), 1.48$ (ddd, $J=13.6,11.7$, $1.9 \mathrm{~Hz}, 1 \mathrm{H}), 1.30-1.16(\mathrm{~m}, 5 \mathrm{H}), 1.18(\mathrm{~d}, J=7.3 \mathrm{~Hz}, 3 \mathrm{H}), 1.13-1.02(\mathrm{~m}, 4 \mathrm{H}), 1.01(\mathrm{~d}, J=7.0 \mathrm{~Hz}$, 3H), $0.98(\mathrm{~d}, J=6.7 \mathrm{~Hz}, 3 \mathrm{H}), 0.92(\mathrm{~d}, J=6.8 \mathrm{~Hz}, 3 \mathrm{H}), 0.89(\mathrm{~d}, J=6.6 \mathrm{~Hz}, 3 \mathrm{H}), 0.80(\mathrm{~d}, J=6.7$ $\mathrm{Hz}, 3 \mathrm{H}), 0.74(\mathrm{~d}, J=6.3 \mathrm{~Hz}, 3 \mathrm{H}) ;{ }^{13} \mathrm{C}$ NMR $\left(125 \mathrm{MHz}, \mathrm{CD}_{3} \mathrm{CN}\right) \delta 173.6,156.9,151.9,133.4$, 132.7 (2C), 132.2, 130.0, 129.9, 129.4, 129.3, 129.0, 128.0, 123.3, 118.3, 117.9, 117.8, (2 carbons obscured by $\left.\mathrm{C}_{6} \mathrm{D}_{6}\right), 115.1,78.7,77.9,76.5,75.0,72.2,62.4,44.6$ (2), 43.0, 42.6, 41.1, $40.1,37.4,36.0,35.4,35.3,35.2,34.4,33.8,33.1,29.3,28.9,25.5,22.9,18.5,17.1,16.4,14.7$, 14.3, 12.1, 8.2; HRMS $\left(\mathrm{ES}^{+}\right) \mathrm{m} / \mathrm{z} 948.542238$ [(M+Na) ${ }^{+}$; calcd for $\left.\mathrm{C}_{51} \mathrm{H}_{79} \mathrm{~N}_{3} \mathrm{NaO}_{10} \mathrm{~S}: 948.538388\right]$. 